\title{
ON DYNAMIC DEVIATION MEASURES AND CONTINUOUS-TIME PORTFOLIO OPTIMIZATION
}

\author{
By Martijn Pistorius ${ }^{1}$ And Mitja Stadje ${ }^{2}$ \\ Imperial College London and University of Ulm
}

\begin{abstract}
In this paper, we propose the notion of dynamic deviation measure, as a dynamic time-consistent extension of the (static) notion of deviation measure. To achieve time-consistency, we require that a dynamic deviation measures satisfies a generalised conditional variance formula. We show that, under a domination condition, dynamic deviation measures are characterised as the solutions to a certain class of stochastic differential equations. We establish for any dynamic deviation measure an integral representation, and derive a dual characterisation result in terms of additively $m$-stable dual sets. Using this notion of dynamic deviation measure, we formulate a dynamic meandeviation portfolio optimization problem in a jump-diffusion setting and identify a subgame-perfect Nash equilibrium strategy that is linear as function of wealth by deriving and solving an associated extended HJB equation.
\end{abstract}

1. Introduction. One traditional way of thinking about risk is in terms of the extend that random realisations deviate from the mean. In portfolio theory as initiated in Markowitz (1952), for instance, risk is quantified as the variance or standard deviation of the return. In the setting of the Black and Scholes (1973) model, it is the volatility parameter, which is equal to the standard deviation of the log-stock price at unit time, that is often taken as description of the risk. Alternative approaches to quantification of risk that have emerged more recently also take into account other aspects of the return distribution such as heavy tails and asymmetry. In this context, an axiomatic framework for (general) deviation measures was introduced and developed in Rockafellar, Uryasev and Zabarankin (2006a), which form a certain class of nonnegative positively homogeneous (static) operators acting on square-integrable random variables. General deviation measures allow to distinguish between upper and lower deviations from the mean, generalising standard deviation. Various aspects of portfolio optimization and financial decision making under general deviation measures have been explored in the literature, in particular regarding CAPM, asset betas, one- and two-fund theorems and equilibrium theory; see also among many others Cheng, Liu and Wang (2004),

Received May 2016; revised January 2017.

${ }^{1}$ Supported in part by EPSRC Grant EP/I019111/1.

${ }^{2}$ Supported by NWO VENI 2012.

MSC2010 subject classifications. 60H30, 90C46, 91A10, 91B70, $93 \mathrm{E} 99$.

Key words and phrases. Deviation measure, time-consistency, portfolio optimization, extended HJB equation. 
Rockafellar, Uryasev and Zabarankin (2006b, 2006c, 2007), Märkert and Schultz (2005), Stoyanov et al. (2008), Grechuk, Molyboha and Zabarankin (2009) or Grechuk and Zabarankin $(2013,2014)$. In this paper we present an axiomatic approach to deviation measures in dynamic continuous-time settings. We show that such dynamic deviation measures admit in general a dual robust representation and are linked to a certain family of stochastic differential equations, if a certain domination condition is satisfied. Subsequently, we use this notion of a dynamic deviation measure to phrase a mean-deviation portfolio optimization problem in a jump-diffusion setting and identify for this problem a subgame-perfect Nash equilibrium portfolio allocation strategy by means of an associated novel type of extended Hamilton-Jacobi-Bellman equation, which complements the ones studied in Björk and Murgoci (2010).

(Conditional) deviation measures. Dynamic deviation measures are given in terms of conditional deviation measures, which are in turn a conditional version of the notion of (static) deviation measure defined in Rockafellar, Uryasev and Zabarankin (2006a) that we describe next. On a filtered probability space $\left(\Omega, \mathcal{F},\left(\mathcal{F}_{t}\right)_{t \in[0, T]}, \mathbb{P}\right)$, where $T>0$ denotes the horizon, consider the (risky) positions described by elements in $L^{p}\left(\mathcal{F}_{t}\right), t \in[0, T], p \geq 0$, the space of $\mathcal{F}_{t}$-measurable random variables $X$ such that $\mathbb{E}\left[|X|^{p}\right]<\infty$. By $L_{+}^{p}\left(\mathcal{F}_{t}\right), L^{\infty}\left(\mathcal{F}_{t}\right)$ and $L_{+}^{\infty}\left(\mathcal{F}_{t}\right)$ are denoted the subsets of nonnegative, bounded and nonnegative bounded elements in $L^{p}\left(\mathcal{F}_{t}\right)$. The definition is given as follows.

Definition 1.1. For any given $t \in[0, T], D_{t}: L^{2}\left(\mathcal{F}_{T}\right) \rightarrow L_{+}^{2}\left(\mathcal{F}_{t}\right)$ is called an $\mathcal{F}_{t}$-conditional deviation measure if it is normalised $\left(D_{t}(0)=0\right)$ and the following properties are satisfied:

(D1) Translation Invariance: $D_{t}(X+m)=D_{t}(X)$ for any $m \in L^{\infty}\left(\mathcal{F}_{t}\right)$.

(D2) Positive Homogeneity: $D_{t}(\lambda X)=\lambda D_{t}(X)$ for any $X \in L^{2}\left(\mathcal{F}_{T}\right)$ and $\lambda \in$ $L_{+}^{\infty}\left(\mathcal{F}_{t}\right)$.

(D3) Subadditivity: $D_{t}(X+Y) \leq D_{t}(X)+D_{t}(Y)$ for any $X, Y \in L^{2}\left(\mathcal{F}_{T}\right)$.

(D4) Positivity: $D_{t}(X) \geq 0$ for any $X \in L^{2}\left(\mathcal{F}_{T}\right)$, and $D_{t}(X)=0$ if and only if $X$ is $\mathcal{F}_{t}$-measurable.

If $\mathcal{F}_{0}$ is trivial, $D_{0}$ is a deviation measure in the sense of Definition 1 in Rockafellar, Uryasev and Zabarankin (2006a). The value $D_{t}(X)=0$, we recall, corresponds to the riskless state of no uncertainty, and axiom (D1) can be interpreted as the requirement that adding to a position $X$ a constant (interpreted as cash) should not increase the risk. Furthermore, it follows similarly as in Rockafellar, Uryasev and Zabarankin (2006a) that, if $D$ satisfies (D2)-(D3), (D1) holds if and only if $D_{t}(m)=0$ for any $m \in L^{2}\left(\mathcal{F}_{t}\right)$. In other words, constants do not carry any risk. Moreover, it is well known that if (D2) holds, (D3) is equivalent 
to conditional convexity, that is, for any $X, Y \in L^{2}\left(\mathcal{F}_{T}\right)$ and any $\lambda \in L^{\infty}\left(\mathcal{F}_{t}\right)$ that is such that $0 \leq \lambda \leq 1$ :

$$
D_{t}(\lambda X+(1-\lambda) Y) \leq \lambda D_{t}(X)+(1-\lambda) D_{t}(Y) .
$$

The property of convexity is often given the interpretation that diversification of a position should not increase its riskiness. We also note that (D2) implies that, for any $X_{1}, X_{2} \in L^{2}\left(\mathcal{F}_{T}\right), D_{t}\left(I_{A} X_{i}\right)=I_{A} D_{t}\left(X_{i}\right), i=1,2$, where $I_{A}$ denotes the indicator of the set $A$, so that ${ }^{3}$

$$
D_{t}\left(I_{A} X_{1}+I_{A^{c}} X_{2}\right)=I_{A} D_{t}\left(X_{1}\right)+I_{A^{c}} D_{t}\left(X_{2}\right), \quad A \in \mathcal{F}_{t} .
$$

In the analysis typically also a lower semicontinuity condition is imposed, the conditional version of which is given as follows:

(D5) Lower Semicontinuity: If $X^{n}$ converges to $X$ in $L^{2}\left(\mathcal{F}_{T}\right)$, then $D_{t}(X) \leq$ $\liminf _{n} D_{t}\left(X^{n}\right)$.

Dynamic deviation measures. We impose additional structure on a given family of $\mathcal{F}_{t}$-conditional deviation measures in order to ensure it satisfies a form of time-consistency. One recursive structure that has been successfully deployed in among others the case of mean-variance portfolio optimization is the one embedded in the conditional variance formula given by $\operatorname{var}_{s}(X)=\operatorname{var}_{s}\left(\mathbb{E}\left[X \mid \mathcal{F}_{t}\right]\right)+$ $\mathbb{E}\left[\operatorname{var}_{t}(X) \mid \mathcal{F}_{s}\right]$ for $s \leq t$ and $X \in L^{2}\left(\mathcal{F}_{T}\right)$ with $\operatorname{var}_{s}(X)=\mathbb{E}\left[\left(X-\mathbb{E}\left[X|| F_{S}\right]\right)^{2} \mid \mathcal{F}_{S}\right]$; see, for instance, Basak and Chabakauri (2010), Wang and Forsyth (2011), Li, Zeng and Lai (2012) or Czichowsky (2013). Inspired by this recursive structure, we require that a collection $\left(D_{t}\right)_{t \in[0, T]}$ of conditional deviation measures satisfy the following generalisation of the conditional variance formula:

(D6) Time-Consistency: For all $s, t \in[0, T]$ with $s \leq t$ and $X \in L^{2}\left(\mathcal{F}_{T}\right)$,

$$
D_{s}(X)=D_{s}\left(\mathbb{E}\left[X \mid \mathcal{F}_{t}\right]\right)+\mathbb{E}\left[D_{t}(X) \mid \mathcal{F}_{s}\right] .
$$

REMARK 1.2. (i) As $D(X) \geq 0$, (D6) implies that $\left(D_{S}(X)\right)_{s \in[0, T]}$ is a supermartingale, which implies in particular that $D$ has a càdlàg modification.

(ii) It follows by standard arguments that (D6) for $s=0$ already uniquely determines a dynamic deviation measure $D$. Suppose that $D_{0}$ and $X \in L^{2}\left(\mathcal{F}_{T}\right)$ are given and besides $\left(D_{t}(X)\right)_{t \in[0, T]}$ there exists a collection of square-integrable $\mathcal{F}_{s}$-measurable random variables $\left(D_{t}^{\prime}(X)\right)_{t \in[0, T]}$ satisfying (D6) for $s=0$, then $D_{t}(X)=D_{t}^{\prime}(X)$ for all $t \in[0, T]$. Indeed, if the $\mathcal{F}_{t}$-measurable set $A^{\prime}:=$ $\left\{D_{t}^{\prime}(X)>D_{t}(X)\right\}$ were to have nonzero measure, then by (1.1) and (D6) we find

$$
\begin{aligned}
\mathbb{E}\left[I_{A^{\prime}} D_{t}(X)\right] & =\mathbb{E}\left[D_{t}\left(I_{A^{\prime}} X\right)\right]=D_{0}\left(I_{A^{\prime}} X\right)-D_{0}\left(\mathbb{E}\left[I_{A^{\prime}} X \mid \mathcal{F}_{t}\right]\right)=\mathbb{E}\left[D_{t}^{\prime}\left(I_{A^{\prime}} X\right)\right] \\
& =\mathbb{E}\left[I_{A^{\prime}} D_{t}^{\prime}(X)\right],
\end{aligned}
$$

\footnotetext{
${ }^{3}$ To see that (1.1) holds, note that by (D2) $I_{A} D_{t}\left(1_{A} X_{1}+1_{A^{c}} X_{2}\right)=D_{t}\left(I_{A}\left(I_{A} X_{1}+1_{A^{c}} X_{2}\right)\right)=$ $D_{t}\left(I_{A} X_{1}\right)=I_{A} D_{t}\left(X_{1}\right)$; similarly, we have $I_{A^{c}} D_{t}\left(1_{A} X_{1}+1_{A^{c}} X_{2}\right)=I_{A^{c}} D_{t}\left(X_{2}\right)$.
} 
which is a contradiction to the definition of the set $A^{\prime}$. Similarly, it may be seen that the set $\left\{D_{t}^{\prime}(X)<D_{t}(X)\right\}$ has measure zero.

(iii) Since $D_{0}$ is convex, lower semicontinuous and finite, $D_{0}$ is continuous in $L^{2}\left(\mathcal{F}_{T}\right)$ [see Proposition 2 in Rockafellar, Uryasev and Zabarankin (2006a, 2006b, 2006c)].

We arrive thus at the following definition of dynamic deviation measure.

DEFINITION 1.3. A family $\left(D_{t}\right)_{t \in[0, T]}$ is called a dynamic deviation measure if $D_{t}, t \in[0, T]$, are $\mathcal{F}_{t}$-conditional deviation measures satisfying (D5) and (D6).

One way to construct examples of dynamic deviation measures is in terms of the solutions of a certain type of SDEs. Such solutions, when seen as function of the corresponding random variable, we will call $g$-deviation measures (where $g$ is the driver function of the SDE in question). We show in Theorem 3.2 that, under a domination condition, any dynamic deviation measure is equal to a $g$-deviation measure for some driver function $g$. This result may be considered to be an analogue of the link between the dynamic coherent and convex risk measures and $g$-expectations; see Coquet et al. (2002) and Royer (2006) [for contributions on convex risk measures and $g$-expectations and their generalizations see, for instance, Barrieu and El Karoui (2005, 2009), Rosazza Gianin (2006), Klöppel and Schweizer (2007), Jiang (2008), El Karoui and Ravanelli (2009), Bion-Nadal and Kervarec (2012) or Pelsser and Stadje (2014)]. By drawing on dual robust representation results, we also establish characterisations of general dynamic deviation measures that are valid without the domination condition (see Theorems 3.7, 3.11 and 3.12).

REMARK 1.4 (Relation to dynamic coherent risk-measures). By generalising arguments given in Rockafellar, Uryasev and Zabarankin (2006a, 2006b, 2006c) to the $\mathcal{F}_{t}$-conditional context, we note that any (static) $\mathcal{F}_{t}$-conditional deviation measure is equal to the sum of a conditional expectation and a risk-measure $\rho_{t}$ that satisfies a $\left(\mathcal{F}_{t}\right.$-conditional) lower range dominance condition. As the notions of time-consistency differ in cases of dynamic deviation and dynamic risk measures this relation does not carry over to the dynamic case (see Proposition 3.14). A collection $\left(\rho_{t}\right)_{t \in[0, T]}, \rho_{t}: L^{2}\left(\mathcal{F}_{T}\right) \rightarrow L_{+}^{2}\left(\mathcal{F}_{t}\right)$, forms a family of dynamic coherent risk measures, we recall, if, for every $t \in[0, T], \rho_{t}$ is positively homogeneous and subadditive [as in (D2) and (D3)], and is (dynamically) monotone and translation invariant in the following sense:

Translation Invariance: For all $X \in L^{2}\left(\mathcal{F}_{T}\right)$ and $m \in L^{\infty}\left(\mathcal{F}_{t}\right)$, we have $\rho_{t}(X+$ $m)=\rho_{t}(X)-m$.

Monotonicity: If $X, Y \in L^{2}\left(\mathcal{F}_{T}\right)$ and $X \leq Y$, then $\rho_{t}(X) \geq \rho_{t}(Y)$. 
For a discussion of these axioms, see Artzner et al. (1999). Note that by (D1)(D2) $D_{t}(m)=0$ for any $m \in L^{2}\left(\mathcal{F}_{t}\right)$, so that dynamic deviation measures do not satisfy the axiom of monotonicity. While for dynamic deviation measures time-consistency is defined in terms of the generalised conditional variance formula (1.2), in the theory of dynamic coherent and convex risk measures a recursive tower-type property is the relation strongly time-consistent dynamic risk-measures should satisfy. Specifically, a dynamic coherent or convex risk measures is called strongly time-consistent, we recall, if

$$
\rho_{s}\left(\rho_{t}(X)\right)=\rho_{s}(X) \quad \text { for } s \leq t ;
$$

see, for instance, among many others Chen and Epstein (2002), Riedel (2004), Delbaen (2006), Artzner et al. (2007), Föllmer and Schied (2011), Cheridito and Kupper (2011). Note that a dynamic deviation measure $D$ is not strongly timeconsistent [in view of the fact that $D_{t}\left(D_{T}(X)\right)=D_{t}(0)=0$ for $t<T$ ]. Interestingly, as shown in Proposition 4.4, a collection of conditional deviation measures satisfies (D6) if and only if in their dual representations the dual sets are convex, closed, and additively $m$-stable, which is a result naturally complementing the well-known fact in the literature that the property of time-consistency for coherent risk measures [defined by (1.3)] may be characterised in terms of convex, closed, multiplicatively $m$-stable sets [see Delbaen (2006)].

Contents. The remainder of the paper is organised as follows. We present in Section 2 the definition of $g$-deviation measures, its properties and a number of examples. With these results in hand, we turn in Section 3.1 to the characterisation of dynamic deviation measures under a domination condition (Theorem 3.2) and proceed to establish in Section 3.2 an integral representation for general dynamic deviation measures, removing the aforementioned domination condition (Theorem 3.7) and a dual robust representation result (Theorems 3.11 and 3.12). In Section 5, we phrase a dynamic mean-deviation portfolio-optimization problem and present an equilibrium solution. It is of interest to investigate other (financial) optimization problems in terms of dynamic deviation measures, such as optimal hedging problems, capital allocation problems and optimal stopping problems; in the interest of brevity, we leave these as topics for future research.

2. $g$-Deviation measures. In the sequel, we assume that the probability space $(\Omega, \mathcal{F}, \mathbb{P})$ is equipped with (i) a standard $d$-dimensional Brownian motion $W=$ $\left(W^{1}, \ldots, W^{d}\right)^{\top}$ and (ii) a Poisson random measure $N(\mathrm{~d} t \times \mathrm{d} x)$ on $[0, T] \times \mathbb{R}^{k} \backslash\{0\}$, independent of $W$, with intensity measure $\hat{N}(\mathrm{~d} t \times \mathrm{d} x)=v(\mathrm{~d} x) \mathrm{d} t$, where the Lévy measure $v(\mathrm{~d} x)$ satisfies the integrability condition

$$
\int_{\mathbb{R}^{k} \backslash\{0\}}\left(|x|^{2} \wedge 1\right) v(\mathrm{~d} x)<\infty,
$$


and let $\tilde{N}(\mathrm{~d} t \times \mathrm{d} x):=N(\mathrm{~d} t \times \mathrm{d} x)-\hat{N}(\mathrm{~d} t \times \mathrm{d} x)$ denote the compensated Poisson random measure. Further, let $\mathcal{U}$ denote the Borel sigma-algebra induced by the $L^{2}(v(\mathrm{~d} x))$-norm, $\left(\mathcal{F}_{t}\right)_{t \in[0, T]}$ the right-continuous completion of the filtration generated by $W$ and $N$, and $\mathcal{P}$ and $\mathcal{O}$ the predictable and optional sigma-algebras on $[0, T] \times \Omega$ with respect to $\left(\mathcal{F}_{t}\right)$. We denote by $L_{d}^{2}(\mathcal{P}, \mathrm{d} \mathbb{P} \times \mathrm{d} t)$ the space of all predictable $d$-dimensional processes that are square-integrable with respect to the measure $\mathrm{d} \mathbb{P} \times \mathrm{d} t$ and we let $\mathcal{S}^{2}=\left\{Y \in \mathcal{O}: \mathbb{E}\left[\sup _{0 \leq t \leq T}\left|Y_{t}\right|^{2}\right]<\infty\right\}$ denote the collection of square-integrable càdlàg optional processes. Further, let $\mathcal{B}\left(\mathbb{R}^{k} \backslash\{0\}\right)$ be the Borel sigma-algebra on $\mathbb{R}^{k} \backslash\{0\}$. For any $X \in L^{2}\left(\mathcal{F}_{T}\right)$ we denote by $\left(H^{X}, \tilde{H}^{X}\right)$ the unique pair of predictable processes with $H^{X} \in L_{d}^{2}(\mathcal{P}, \mathrm{d} \mathbb{P} \times \mathrm{d} t)$ and $\tilde{H}^{X} \in L^{2}\left(\mathcal{P} \times \mathcal{B}\left(\mathbb{R}^{k} \backslash\{0\}\right), \mathrm{d} \mathbb{P} \times \mathrm{d} t \times v(\mathrm{~d} x)\right)$, subsequently referred to as the representing pair of $X$, satisfying ${ }^{4}$

$$
X=\mathbb{E}[X]+\int_{0}^{T} H_{s}^{X} \mathrm{~d} W_{s}+\int_{0}^{T} \int_{\mathbb{R}^{k} \backslash\{0\}} \tilde{H}_{s}^{X}(x) \tilde{N}(\mathrm{~d} s \times \mathrm{d} x),
$$

where $\int_{0}^{T} H_{s}^{X} \mathrm{~d} W_{s}:=\sum_{i=1}^{d} \int_{0}^{T} H_{s}^{X, i} \mathrm{~d} W_{s}^{i}$.

We consider the following class of driver functions.

DEFINITION 2.1. We call a $\mathcal{P} \otimes \mathcal{B}\left(\mathbb{R}^{d}\right) \otimes \mathcal{U}$-measurable function

$$
\begin{aligned}
g:[0, T] \times \Omega \times \mathbb{R}^{d} \times L^{2}(v(\mathrm{~d} x)) & \rightarrow \mathbb{R}_{+}, \\
(t, \quad \omega, \quad h, \quad \tilde{h}) \quad & \longmapsto g(t, \omega, h, \tilde{h})
\end{aligned}
$$

a driver function if for $\mathrm{dP} \times \mathrm{d} t$ a.e. $(\omega, t) \in \Omega \times[0, T]$ :

(i) (Positivity.) For any $(h, \tilde{h}) \in \mathbb{R}^{d} \times L^{2}(v(\mathrm{~d} x)) g(t, h, \tilde{h}) \geq 0$ with equality if and only if $(h, \tilde{h})=0$.

(ii) (Lower semicontinuity.) If $h^{n} \rightarrow h, \tilde{h}^{n} \rightarrow \tilde{h} L^{2}(v(\mathrm{~d} x))$-a.e., then $g(t, h, \tilde{h}) \leq \liminf _{n} g\left(t, h^{n}, \tilde{h}^{n}\right)$.

DEFINITION 2.2. We call a driver function $g$ convex if $g(t, h, \tilde{h})$ is convex in $(h, \tilde{h}), \mathrm{d} \mathbb{P} \times \mathrm{d} t$ a.e.; positively homogeneous if $g(t, h, \tilde{h})$ is positively homogeneous in $(h, \tilde{h})$, that is, for $\lambda>0, g(t, \lambda h, \lambda \tilde{h})=\lambda g(t, h, \tilde{h}), \mathrm{d} \mathbb{P} \times \mathrm{d} t$ a.e. and of linear growth if for some $K>0$ we have $\mathrm{d} \mathbb{P} \times \mathrm{d} t$ a.e.,

$$
|g(t, h, \tilde{h})|^{2} \leq 1+K^{2}|h|^{2}+K^{2} \int_{\mathbb{R}^{k} \backslash\{0\}} \tilde{h}(x)^{2} v(\mathrm{~d} x) .
$$

To such a driver function $g$, one may associate a corresponding dynamic deviation measure given in terms of the solution to a certain (backward) SDE.

\footnotetext{
${ }^{4}$ See, for example, Theorem III.4.34 in Jacod and Shiryaev (2003).
} 
DEFINITION 2.3. Let $g$ be a convex and positively homogeneous driver function of linear growth. The $g$-deviation measure $D^{g}=\left(D_{t}^{g}\right)_{t \in[0, T]}$ is equal to the collection $D_{t}: L^{2}\left(\mathcal{F}_{T}\right) \rightarrow L_{+}^{2}\left(\mathcal{F}_{t}\right), t \in[0, T]$, given by

$$
D_{t}^{g}(X)=Y_{t}, \quad X \in L^{2}\left(\mathcal{F}_{T}\right),
$$

where $(Y, Z, \tilde{Z}) \in \mathcal{S}^{2} \times L_{d}^{2}(\mathcal{P}, \mathrm{d} \mathbb{P} \times \mathrm{d} t) \times L^{2}\left(\mathcal{P} \times \mathcal{B}\left(\mathbb{R}^{k} \backslash\{0\}\right), \mathrm{d} \mathbb{P} \times \mathrm{d} t \times v(\mathrm{~d} x)\right)$ is the unique solution of the SDE given in terms of the representing pair $\left(H^{X}, \tilde{H}^{X}\right)$ of $X$ by

$$
\begin{aligned}
\mathrm{d} Y_{t}= & -g\left(t, H_{t}^{X}, \tilde{H}_{t}^{X}\right) \mathrm{d} t+Z_{t} \mathrm{~d} W_{t} \\
& +\int_{\mathbb{R}^{k} \backslash\{0\}} \tilde{Z}_{t}(x) \tilde{N}(\mathrm{~d} t \times \mathrm{d} x), \quad t \in[0, T), \\
Y_{T}= & 0,
\end{aligned}
$$

Any $g$-deviation measure admits an integral representation in terms of $g$.

PROPOSITION 2.4. Let $g$ be a convex and positively homogeneous driver function of linear growth.

(i) For given $X \in L^{2}\left(\mathcal{F}_{T}\right)$, we have

$$
D_{t}^{g}(X)=\mathbb{E}\left[\int_{t}^{T} g\left(s, H_{s}^{X}, \tilde{H}_{s}^{X}\right) \mathrm{d} s \mid \mathcal{F}_{t}\right], \quad t \in[0, T] .
$$

(ii) $D^{g}$ is a dynamic deviation measure. In particular, $D^{g}$ satisfies (D6).

PROOF. (i) Letting $Y_{t}$ be equal to the right-hand side of (2.5) we note that $Y_{T}=0$, while we have

$$
Y_{t}=M_{t}-\int_{0}^{t} g\left(s, H_{s}^{X}, \tilde{H}_{s}^{X}\right) \mathrm{d} s, \quad M_{t}=\mathbb{E}\left[\int_{0}^{T} g\left(s, H_{s}^{X}, \tilde{H}_{s}^{X}\right) \mathrm{d} s \mid \mathcal{F}_{t}\right] .
$$

Letting $(Z, \tilde{Z})=\left(Z^{M_{T}}, \tilde{Z}^{M_{T}}\right)$ the representing pair of $M_{T}$ we have that $Y_{t}$ satisfies (2.3).

(ii) To verify that (D6) holds, we note that the representation (2.5) implies that, for any $s, t \in[0, T]$ with $s \leq t$,

$$
D_{s}^{g}\left(\mathbb{E}\left[X \mid \mathcal{F}_{t}\right]\right)=\mathbb{E}\left[\int_{s}^{t} g\left(u, H_{u}^{X}, \tilde{H}_{u}^{X}\right) \mathrm{d} u \mid \mathcal{F}_{s}\right],
$$

which yields that $D_{s}^{g}\left(\mathbb{E}\left[X \mid \mathcal{F}_{t}\right]\right)+\mathbb{E}\left[D_{t}^{g}(X) \mid \mathcal{F}_{s}\right]$ is equal to

$$
\begin{gathered}
\mathbb{E}\left[\int_{s}^{t} g\left(u, H_{u}^{X}, \tilde{H}_{u}^{X}\right) \mathrm{d} u \mid \mathcal{F}_{s}\right]+\mathbb{E}\left[\mathbb{E}\left[\int_{t}^{T} g\left(u, H_{u}^{X}, \tilde{H}_{u}^{X}\right) \mathrm{d} u \mid \mathcal{F}_{t}\right] \mid \mathcal{F}_{s}\right] \\
\quad=\mathbb{E}\left[\int_{s}^{T} g\left(u, H_{u}^{X}, \tilde{H}_{u}^{X}\right) \mathrm{d} u \mid \mathcal{F}_{s}\right],
\end{gathered}
$$


which is equal to $D_{S}^{g}(X)$. We show next that the axioms (D1)-(D5) are satisfied. We note from $(2.5)$ that $D_{t}^{g}(X+m)=D_{t}^{g}(X)$ for any $X \in L^{2}\left(\mathcal{F}_{T}\right), m \in L_{+}^{\infty}\left(\mathcal{F}_{t}\right)$ while $D_{t}^{g}(m)=0$ as $g(t, 0,0)=0$, so that (D1) holds. Using (2.5), we see that $D^{g}$ inherits the properties of convexity and positive homogeneity from $g$, so that (D2) and (D3) are satisfied. Positivity (D4) is straightforward to verify by using that $g$ is nonnegative and strictly positive for $(h, \tilde{h}) \neq 0$. Finally, noting that (a) if $X^{n} \rightarrow X$ in $L^{2}\left(\mathcal{F}_{T}\right),\left(H^{X^{n}}, \tilde{H}^{X^{n}}\right)$ converges to $\left(H^{X}, \tilde{H}^{X}\right)$ in $L_{d}^{2}(\mathrm{~d} \mathbb{P} \times \mathrm{d} t) \times L^{2}(\mathrm{~d} \mathbb{P} \times \mathrm{d} t \times$ $v(\mathrm{~d} x)$ )-norm and that (b) $g$ is nonnegative and lower semicontinuous, we have by an application of Fatou's lemma

$$
\begin{aligned}
\liminf _{n} D_{t}^{g}\left(X^{n}\right) & =\liminf _{n} \mathbb{E}\left[\int_{t}^{T} g\left(s, H_{s}^{X^{n}}, \tilde{H}_{s}^{X^{n}}\right) \mathrm{d} s \mid \mathcal{F}_{t}\right] \\
& \geq \mathbb{E}\left[\int_{t}^{T} \liminf _{n} g\left(s, H_{s}^{X^{n}}, \tilde{H}_{s}^{X^{n}}\right) \mathrm{d} s \mid \mathcal{F}_{t}\right] \\
& \geq \mathbb{E}\left[\int_{t}^{T} g\left(s, H_{s}^{X}, \tilde{H}_{s}^{X}\right) \mathrm{d} s \mid \mathcal{F}_{t}\right] \\
& =D_{t}^{g}(X),
\end{aligned}
$$

which shows that also the lower semicontinuity condition in (D5) is satisfied.

The linear growth condition and convexity guarantee that a $g$-deviation measure is continuous in $L^{2}$.

LEMMA 2.5. Let $g$ be a convex driver function of linear growth. If $X^{n}$ converge to $X$ in $L^{2}\left(\mathcal{F}_{T}\right)$, then $\lim _{n} D_{0}^{g}\left(X^{n}\right)=D_{0}^{g}(X)$.

Proof. If $X^{n}$ converge to $X$ in $L^{2}\left(\mathcal{F}_{T}\right)$ then, as noted before, $H^{X^{n}}$ and $\tilde{H}^{X^{n}}$ converge to $H^{X}$ and $\tilde{H}^{X}$ in $L_{d}^{2}(\mathrm{~d} \mathbb{P} \times \mathrm{d} t)$ and $L^{2}(\mathrm{~d} \mathbb{P} \times \mathrm{d} t \times v(\mathrm{~d} x))$ norms. Next, note that $\left|g\left(s, H_{s}^{X^{n}}, \tilde{H}_{S}^{X^{n}}\right)\right|$ is a uniformly integrable sequence by the growth-condition (2.2) and the convergence of the processes $\left|H^{X^{n}}\right|^{2}$ and $\int_{\mathbb{R}^{k} \backslash\{0\}}\left|\tilde{H}^{X^{n}}\right|^{2}(x) v(\mathrm{~d} x)$ in $L^{1}(\mathrm{~d} \mathbb{P} \times \mathrm{d} t)$-norm. As $g$ is continuous [as it is convex and locally bounded, $c f$. Theorem 2.2.9 in Zălinescu (2002)], it follows thus that $\lim _{n} D_{0}^{g}\left(X^{n}\right)=\lim _{n} \mathbb{E}\left[\int_{0}^{T} g\left(s, H_{s}^{X^{n}}, \tilde{H}_{s}^{X^{n}}\right) \mathrm{d} s\right]=\mathbb{E}\left[\int_{0}^{T} g\left(s, H_{s}^{X}, \tilde{H}_{s}^{X}\right) \mathrm{d} s\right]=$ $D_{0}^{g}(X)$.

We list a number of properties of a $g$-deviation measure that are characterised in terms of those of the driver function $g$.

PROPOSITION 2.6. Let $g$ and $\tilde{g}$ be driver functions of linear growth:

(i) $D^{g}$ is conditionally convex if and only if $g$ is convex.

(ii) $D^{g}$ satisfies (D2) if and only if $g$ is positively homogeneous. 
(iii) $D^{g}$ is symmetric, that is, $D_{t}^{g}(X)=D_{t}^{g}(-X)$ for all $t$, if and only if $g$ is symmetric in $(h, \tilde{h})$.

(iv) $D^{g} \geq D^{\tilde{s}}$ if and only if $g \geq \tilde{g} \mathrm{~d} \mathbb{P} \times \mathrm{d} t$ a.e.

To simplify notation, we denote, for $s, t \in[0, T]$ with $s \leq t$ and $(H, \tilde{H}) \in$ $L_{d}^{2}\left(\mathcal{P}, \mathrm{d} \mathbb{P} \times \mathrm{d} t \times L^{2}\left(\mathcal{P} \times \mathcal{B}\left(\mathbb{R}^{k} \backslash\{0\}\right), \mathrm{d} \mathbb{P} \times \mathrm{d} t \times v(\mathrm{~d} x)\right),(H \cdot W)_{s, t}:=\int_{s}^{t} H_{u} \mathrm{~d} W_{u}\right.$ and $(\tilde{H} \cdot \tilde{N})_{s, t}:=\int_{(s, t] \times \mathbb{R}^{k} \backslash\{0\}} \tilde{H}_{u}(x) \tilde{N}(\mathrm{~d} u \times \mathrm{d} x)$, and moreover, $(H \cdot W)_{t}:=$ $(H \cdot W)_{0, t}$ and $(\tilde{H} \cdot \tilde{N})_{t}:=(\tilde{H} \cdot \tilde{N})_{0, t}$.

ProOF OF Proposition 2.6. First, we prove (i) " $\Rightarrow$ " by contradiction. Suppose that there exist predictable processes $B^{i}$ and $\widetilde{B}^{i}$ for $i=1,2$, a nonzero predictable set $C$ and a $\lambda \in(0,1)$ such that for $(s, \omega) \in C$

$$
\begin{aligned}
& g\left(s, \lambda B_{s}^{1}+(1-\lambda) B_{s}^{2}, \lambda \tilde{B}_{s}^{1}+(1-\lambda) \tilde{B}_{s}^{2}\right) \\
& \quad>\lambda g\left(s, B_{s}^{1}, \tilde{B}_{s}^{1}\right)+(1-\lambda) g\left(s, B_{s}^{2}, \tilde{B}_{s}^{2}\right) .
\end{aligned}
$$

Set $H_{s}^{i}(\omega)=B_{s}^{i}(\omega), i=1,2$, if $(s, \omega) \in C$ and zero otherwise, define $\tilde{H}^{i}, i=1,2$, similarly and set $X=\left(H^{1} \cdot W\right)_{T}+\left(\tilde{H}^{1} \cdot \tilde{N}\right)_{T}, Y=\left(H^{2} \cdot W\right)_{T}+\left(\tilde{H}^{2} \cdot \tilde{N}\right)_{T}$ and $C_{s}=\{\omega \in \Omega:(s, \omega) \in C\}$. using that $g(s, 0,0)=0$ it follows that $D_{0}^{g}(\lambda X+(1-$ $\lambda) Y$ ) is equal to

$$
\begin{aligned}
\mathbb{E}\left[\int_{0}^{T} g\left(s, \lambda I_{C_{s}} H_{s}^{1}+(1-\lambda) I_{C_{s}} H_{s}^{2}, \lambda I_{C_{s}} \tilde{H}_{s}^{1}+(1-\lambda) I_{C_{s}} \tilde{H}_{s}^{2}\right) \mathrm{d} s\right] \\
=\mathbb{E}\left[\int_{0}^{T} I_{C_{s}} g\left(s, \lambda H_{s}^{1}+(1-\lambda) H_{s}^{2}, \lambda \tilde{H}_{s}^{1}+(1-\lambda) \tilde{H}_{s}^{2}\right) \mathrm{d} s\right] \\
>\lambda \mathbb{E}\left[\int_{0}^{T} I_{C_{s}} g\left(s, H_{s}^{1}, \tilde{H}_{s}^{1}\right) \mathrm{d} s\right]+(1-\lambda) \mathbb{E}\left[\int_{0}^{T} I_{C_{s}} g\left(s, H_{s}^{2}, \tilde{H}_{s}^{2}\right) \mathrm{d} s\right] \\
=\lambda \mathbb{E}\left[\int_{0}^{T} g\left(s, I_{C_{s}} H_{s}^{1}, I_{C_{s}} \tilde{H}_{s}^{1}\right) \mathrm{d} s\right] \\
\quad+(1-\lambda) \mathbb{E}\left[\int_{0}^{T} g\left(s, I_{C_{s}} H_{s}^{2}, I_{C_{s}} \tilde{H}_{s}^{2}\right) \mathrm{d} s\right] .
\end{aligned}
$$

The right-hand side of (2.6) is equal to $\lambda D_{0}^{g}(X)+(1-\lambda) D_{0}^{g}(Y)$, in contradiction to the convexity of $D_{0}^{g}$. The directions " $\Rightarrow$ " in (ii), (iii) and (iv) follow by similar lines of reasoning. The implications " $\Leftarrow$ " in (i)-(iv) follow from (2.5) in Proposition 2.4.

Examples. We give next a number of examples of $g$-deviation measures.

EXAMPLE 2.7. The family of $g$-deviation measures with driver functions given by

$$
g_{c, d}(t, h, \tilde{h})=c|h|+d \sqrt{\int_{\mathbb{R}^{k} \backslash\{0\}}|\tilde{h}(x)|^{2} v(\mathrm{~d} x)}, \quad c, d \in \mathbb{R}_{+} \backslash\{0\},
$$


corresponds to a measurement of the risk of a random variable $X \in L^{2}\left(\mathcal{F}_{T}\right)$ by the integrated multiples of the local volatilities of the continuous and discontinuous martingale parts in its martingale representation (2.1).

EXAMPLE 2.8. In the case of a $g$-deviation measure with driver function given by

$$
g(\omega, t, h, \tilde{h})=\operatorname{CVaR}_{t, a}^{v}(\tilde{h}), \quad a \in\left(0, v\left(\mathbb{R}^{k} \backslash\{0\}\right)\right),
$$

the risk is measured in terms of the values of the (large) jump sizes under $\mathrm{CVaR}_{t, a}^{v}$. Here $\operatorname{CVaR}_{t, a}^{v}(\tilde{h})=\frac{1}{a} \int_{0}^{a} \operatorname{VaR}_{t, b}^{v}(\tilde{h}) \mathrm{d} b$ is given in terms of the leftquantiles $\operatorname{VaR}_{t, a}^{v}(\tilde{h}), a \in\left(0, v\left(\mathbb{R}^{k} \backslash\{0\}\right)\right)$ of $h(J)$ under the measure $v(\mathrm{~d} x)$, that is,

$\operatorname{VaR}_{t, a}^{v}(\tilde{h}):=\operatorname{VaR}_{a}^{v}(h(J)):=\sup \left\{y \in \mathbb{R}: v\left(\left\{x \in \mathbb{R}^{k} \backslash\{0\}: \tilde{h}(x)<-y\right\}\right)<a\right\}$.

In the next example, we deploy the following auxiliary result.

PROPOSITION 2.9. Let $I:=\left\{t_{0}, t_{1}, \ldots, t_{n}\right\} \subset[0, T]$ be strictly ordered. $D=\left(D_{t}\right)_{t \in I}$ satisfies (D1)-(D4) and (D6) if and only if for some collection $\tilde{D}=\left(\tilde{D}_{t}\right)_{t \in I}$ of conditional deviation measures we have

$$
D_{t}(X)=\mathbb{E}\left[\sum_{t_{i} \in I: t_{i} \geq t} \tilde{D}_{t_{i}}\left(\mathbb{E}\left[X \mid \mathcal{F}_{t_{i+1}}\right]-\mathbb{E}\left[X \mid \mathcal{F}_{t_{i}}\right]\right) \mid \mathcal{F}_{t}\right],
$$

$$
t \in I, X \in L^{2}\left(\mathcal{F}_{T}\right) .
$$

In particular, a dynamic deviation measure $D$ satisfies (2.8) with $\tilde{D}_{t_{i}}=D_{t_{i}}, t_{i} \in I$.

PROOF. " $\Leftarrow ":$ We will only show that $D_{t}$ satisfies (D6), as it is clear that (D1)-(D4) are satisfied. Let $X \in L^{2}\left(\mathcal{F}_{T}\right)$ and note that as $\tilde{D}_{t}, t \in I$, satisfy (D1) and (D4) we have for any $s, t \in I$ with $s>t$ that $D_{t}\left(\mathbb{E}\left[X \mid \mathcal{F}_{s}\right]\right)=$ $\sum_{t_{i} \in I: t \leq t_{i}<s} \mathbb{E}\left[\tilde{D}_{t_{i}}\left(\mathbb{E}\left[X \mid \mathcal{F}_{t_{i+1}}\right]\right) \mid \mathcal{F}_{t}\right]$. Thus, we have that $D_{t}(X)$ is equal to

$$
\begin{aligned}
& \sum_{t_{i} \in I: t \leq t_{i}<s} \mathbb{E}\left[\tilde{D}_{t_{i}}\left(\mathbb{E}\left[X \mid \mathcal{F}_{t_{i+1}}\right]\right) \mid \mathcal{F}_{t}\right]+\sum_{t_{i} \in I: s \leq t_{i}} \mathbb{E}\left[\tilde{D}_{t_{i}}\left(\mathbb{E}\left[X \mid \mathcal{F}_{t_{i+1}}\right]\right) \mid \mathcal{F}_{t}\right] \\
& \quad=D_{t}\left(\mathbb{E}\left[X \mid \mathcal{F}_{s}\right]\right)+\mathbb{E}\left[D_{s}(X) \mid \mathcal{F}_{t}\right] .
\end{aligned}
$$

“ $\Rightarrow$ ": For $X \in L^{2}\left(\mathcal{F}_{T}\right)$ and $t_{i-1} \in I, i \geq 1$, we have by (D6) and (D1)

$$
\begin{aligned}
D_{t_{i-1}}(X) & =D_{t_{i-1}}\left(\mathbb{E}\left[X \mid \mathcal{F}_{t_{i}}\right]\right)+\mathbb{E}\left[D_{t_{i}}(X) \mid \mathcal{F}_{t_{i-1}}\right] \\
& =D_{t_{i-1}}\left(\mathbb{E}\left[X \mid \mathcal{F}_{t_{i}}\right]-\mathbb{E}\left[X \mid \mathcal{F}_{t_{i-1}}\right]\right)+\mathbb{E}\left[D_{t_{i}}(X) \mid \mathcal{F}_{t_{i-1}}\right]
\end{aligned}
$$

An induction argument based on (2.9) then yields that (2.8) holds with $\tilde{D}_{t}=D_{t}$, $t \in I$. 
EXAMPLE 2.10. The formula (2.8) in Proposition 2.9 gives a way to define a collection $D=\left(D_{t}\right)_{t \in I}$ satisfying axioms (D1)-(D6) for $s, t \in I$, which we call a dynamic deviation measure on the grid I. Comparison of (2.8) and (2.5) suggests that one may obtain the values of a dynamic deviation measure as limit of the values of (suitably chosen) dynamic deviation measures on grids with vanishing mesh sizes. We next illustrate this for the $g$-deviation measures $\bar{D}^{\lambda}:=D^{g_{\lambda}}, \lambda>0$, corresponding to the driver functions $g_{\lambda}$ given by

$$
g_{\lambda}(\omega, t, h, \tilde{h}):=\lambda \sqrt{|h|^{2}+\int_{\mathbb{R}^{k} \backslash\{0\}}|\tilde{h}(x)|^{2} v(\mathrm{~d} x)}, \quad \lambda>0,
$$

and random variables $X \in L^{2}\left(\mathcal{F}_{T}\right)$ of the form

$$
X=x+\int_{0}^{T} f(t) \mathrm{d} W_{t}+\int_{[0, T] \times \mathbb{R}^{k} \backslash\{0\}} g(t, y) \tilde{N}(\mathrm{~d} t \times \mathrm{d} y)
$$

with $x \in \mathbb{R}, f \in C\left([0, T], \mathbb{R}^{d}\right)$ and $g \in C_{0}\left([0, T] \times \mathbb{R}^{k}, \mathbb{R}\right){ }^{5}$ We construct approximating sequences in terms of the conditional CVaR-deviation measures given by $\tilde{D}_{t}(Y):=\mathrm{CVaR}_{t, \alpha}\left(Y-\mathbb{E}\left[Y \mid \mathcal{F}_{t}\right]\right)$ for $Y \in L^{2}\left(\mathcal{F}_{T}\right), t \in[0, T], \alpha \in(0,1)$, where for $Z \in L^{2}\left(\mathcal{F}_{T}\right)$

$$
\begin{aligned}
\operatorname{CVaR}_{t, \alpha}(Z) & =\frac{1}{\alpha} \int_{0}^{\alpha} \operatorname{VaR}_{t, b}(Z) \mathrm{d} b, \\
\operatorname{VaR}_{t, b}(Z) & =\sup \left\{y \in \mathbb{R}: \mathbb{P}\left(Z<-y \mid \mathcal{F}_{t}\right)<b\right\} ;
\end{aligned}
$$

see Rockafellar, Uryasev and Zabarankin (2006a).

Specifically, the expression in (2.8) suggests to scale the value of conditional deviation measures corresponding to small time units in order to obtain in the limit a dynamic deviation measure. Denoting for $X$ of the form (2.11),

$$
\begin{aligned}
M_{t_{i+1}}:=\mathbb{E}\left[X \mid \mathcal{F}_{t_{i+1}}\right], \quad \Delta M_{i+1}:= & M_{t_{i+1}}-M_{t_{i}}, \\
& t_{i}=T i / 2^{n}, i=0, \ldots, 2^{n}-1,
\end{aligned}
$$

with $t_{2^{n}}=T$ and following this suggestion we specify the contribution to the total risk of

$$
\begin{aligned}
\Delta M_{i+1}=\int_{t_{i}}^{t_{i+1}} f(s) \mathrm{d} W_{s}+\int_{\left(t_{i}, t_{i+1}\right] \times\left(\mathbb{R}^{k} \backslash\{0\}\right)} g(s, y) \tilde{N}(\mathrm{~d} s \times \mathrm{d} y), & \\
i & =0, \ldots, 2^{n}-1,
\end{aligned}
$$

${ }^{5} C\left([0, T], \mathbb{R}^{d}\right)$ and $C_{0}\left([0, T] \times \mathbb{R}^{k}, \mathbb{R}\right)$ denote the sets of continuous functions $f:[0, T] \mapsto \mathbb{R}^{d}$, and of continuous functions $g:[0, T] \times \mathbb{R}^{k} \mapsto \mathbb{R}$ that are such that $\sup _{t \in[0, T]}|g(t, x)| \rightarrow 0$ as $|x| \rightarrow$ $\infty$ and $\sup _{x \in \mathbb{R}^{k} \backslash\{0\}} \sup _{t \in[0, T]}\left\{|g(t, x)| /|x|^{2}\right\}<\infty$. 
by $\tilde{D}_{t_{i}}\left(\Delta M_{i+1}\right):=\sqrt{\Delta t_{i+1}} \mathrm{CVaR}_{t_{i}, \alpha}\left(\Delta M_{i+1}\right), \Delta t_{i+1}=t_{i+1}-t_{i}$, which gives rise to the dynamic deviation measure $D^{(n)}=\left(D_{t}^{(n)}\right)_{t \in I_{n}}$ on $I_{n}:=\left\{t_{i}, i=0, \ldots, 2^{n}\right\}$ given by

$$
\begin{aligned}
D_{t}^{(n)}(X)= & \sum_{t_{i} \geq t} \mathbb{E}\left[\tilde{D}_{t_{i}}\left(\Delta M_{i+1}\right) \mid \mathcal{F}_{t}\right] \\
= & \sum_{t_{i} \geq t} \sqrt{\sigma^{2}\left(t_{i}\right)} \Delta t_{i+1} \mathbb{E}\left[\operatorname{CVaR}_{t_{i}, \alpha}\left(\frac{\Delta M_{i+1}}{\sqrt{\sigma^{2}\left(t_{i}\right) \Delta t_{i+1}}}\right) \mid \mathcal{F}_{t}\right], \\
& \text { with } \sigma^{2}(t):=|f(t)|^{2}+\int_{\mathbb{R}^{k} \backslash\{0\}}|g(t, x)|^{2} v(\mathrm{~d} x), t \in I_{n},
\end{aligned}
$$

where we used that $\mathrm{CVaR}_{t_{i}, \alpha}$ is positively homogeneous. As $\Delta M_{i+1}$ is infinitely divisible and $f$ and $g$ are bounded, we have by an application of the LindebergFeller central limit theorem [see, e.g., Durrett (2010), p. 129] that, when we let $n \rightarrow \infty$ while keeping $t_{i}$ fixed the ratio $\Delta M_{i+1} / \sqrt{\sigma^{2}\left(t_{i}\right) \Delta t_{i+1}}$ converges in distribution to a standard normal random variable $\xi$. By uniform integrability and the independence of $\Delta M_{i+1}$ from $\mathcal{F}_{t_{i}}$, we have

$$
\begin{aligned}
\operatorname{CVaR}_{\alpha, t_{i}}\left(\frac{\Delta M_{i+1}}{\sqrt{\sigma^{2}\left(t_{i}\right) \Delta t_{i+1}}}\right) & =\mathrm{CVaR}_{\alpha}\left(\frac{\Delta M_{i+1}}{\sqrt{\sigma^{2}\left(t_{i}\right) \Delta t_{i+1}}}\right) \rightarrow \operatorname{CVaR}_{\alpha}(\xi) \\
& =\frac{1}{\alpha} \int_{0}^{\alpha} \Phi^{-1}(u) \mathrm{d} u=: c_{\alpha},
\end{aligned}
$$

where $\operatorname{CVaR}_{\alpha}(\cdot)=\operatorname{CVaR}_{\alpha, 0}(\cdot)$ and $\Phi^{-1}$ denotes the inverse of the standard normal distribution function $\Phi$. Hence, letting $n \rightarrow \infty$ in (2.12) and deploying the uniform continuity of $f$ and $g$ we have for any $t \in[0, T]$ of the form $t=k / 2^{m}, k, m \in \mathbb{N}$,

$$
\begin{aligned}
D_{t}^{(n)}(X) & \rightarrow c_{\alpha} \mathbb{E}\left[\int_{t}^{T} \sqrt{|f(s)|^{2}+\int_{\mathbb{R}^{k} \backslash\{0\}}|g(t, x)|^{2} v(\mathrm{~d} x)} \mathrm{d} s \mid \mathcal{F}_{t}\right] \\
& =\bar{D}_{t}^{c_{\alpha}}(X) .
\end{aligned}
$$

\section{Representation results, $m$-stability and time-consistency.}

3.1. Characterisation result. We show next that any dynamic deviation measure that satisfies a domination condition is a $g$-deviation measure for some driver function $g$.

Definition 3.1. A dynamic deviation measure $D=\left(D_{t}\right)_{t \in[0, T]}$ is called $\lambda$ dominated if for all $t \in[0, T]$ and $X \in L^{2}\left(\mathcal{F}_{T}\right)$ and we have

$$
D_{t}(X) \leq \bar{D}_{t}^{\lambda}(X)
$$


Proposition 3.2. Let $D=\left(D_{t}\right)_{t \in[0, T]}$ be a collection of maps $D_{t}: L^{2}\left(\mathcal{F}_{T}\right) \rightarrow L_{+}^{2}\left(\mathcal{F}_{t}\right), t \in[0, T]$. Then $D$ is a dynamic deviation measure that is $\lambda$-dominated for some $\lambda>0$ if and only if there exists a convex and positively homogeneous driver function $g$ of linear growth such that $D=D^{g}$. Furthermore, this driver function $g$ is unique $\mathrm{d} \mathbb{P} \times \mathrm{d} t$ a.e.

PROOF. We first verify uniqueness: If $\bar{g}$ is a driver function that satisfies $D^{g}=$ $D^{\bar{g}}$, it follows from Proposition 2.6(iv) that $g=\bar{g} \mathrm{~d} \mathbb{P} \times \mathrm{d} t$ a.e. We note next that the implication " $\Leftarrow$ " follows from Proposition 2.4. The remainder is devoted to the proof of the implication " $\Rightarrow$ ", which is established using a number of auxiliary results (the proofs of which are deferred to the end of the section).

Thus, let $D$ be a given dynamic deviation measure that is $\lambda$-dominated, so that in particular $D_{0}$ is finite. We identify next a candidate driver function $g$. For the remainder of the proof, we assume for the ease of presentation that $d=1$. For fixed $h \in \mathbb{R}$ and $\tilde{h} \in L^{2}(v(\mathrm{~d} x))$, consider the mapping $\mu_{h, \tilde{h}}: \mathcal{P} \times \mathcal{P} \rightarrow \mathbb{R}$ given by

$$
\mu_{h, \tilde{h}}: C_{1} \times C_{2} \mapsto D_{0}\left(\left(I_{C_{1}} h \cdot W\right)_{T}+\left(I_{C_{2}} \tilde{h} \cdot \tilde{N}\right)_{T}\right) .
$$

LEMmA 3.3. Let $(h, \tilde{h}) \in \mathbb{R} \times L^{2}(v(\mathrm{~d} x))$.

(i) $C \mapsto \mu_{h, \tilde{h}}(C, \varnothing), C \mapsto \mu_{h, \tilde{h}}(\varnothing, C)$ and $C \mapsto \mu_{h, \tilde{h}}(C, C)$ are $\sigma$-finite measures on $([0, T] \times \Omega, \mathcal{P})$.

(ii) For any $C_{1}, C_{2} \in \mathcal{P}$ we have

$$
\begin{aligned}
\mu_{h, \tilde{h}}\left(C_{1}, C_{2}\right)= & \mu_{h, \tilde{h}}\left(C_{1} \backslash C_{2}, \varnothing\right)+\mu_{h, \tilde{h}}\left(\varnothing, C_{2} \backslash C_{1}\right) \\
& +\mu_{h, \tilde{h}}\left(C_{1} \cap C_{2}, C_{1} \cap C_{2}\right) .
\end{aligned}
$$

The proof of Lemma 3.3 is given in the Appendix. As $D_{0}$ is $\lambda$-dominated $C \mapsto \mu_{h, \tilde{h}}(C, C)$ is absolutely continuous with respect to the measure $\mathrm{d} \mathbb{P} \times \mathrm{d} t$ and we conclude from the Radon-Nikodym theorem that there exists an integrable nonnegative density, say $R_{h, \tilde{h}}(s, \omega)$, that is such that $R_{0,0}=0$ and for any set $C \in \mathcal{P}$

$$
\mu_{h, \tilde{h}}(C, C)=\mathbb{E}\left[\int_{0}^{T} I_{C_{s}} R_{h, \tilde{h}}(s) \mathrm{d} s\right],
$$

where $C_{s}=\{\omega \in \Omega:(\omega, s) \in C\}$. In particular, we note that $\mu_{h, \tilde{h}}(C, \varnothing)=$ $\mu_{h, 0}(C, C)$ and $\mu_{h, \tilde{h}}(\varnothing, C)=\mu_{0, \tilde{h}}(C, C)$ satisfy (3.2) with $R_{h, \tilde{h}}$ replaced by $R_{0, \tilde{h}}$ and $R_{h, 0}$, respectively. We define the candidate driver function $g$ in terms of $R$ by

$$
g(t, \omega, h, \tilde{h}):=R_{h, \tilde{h}}(t, \omega), \quad(t, \omega) \in[0, T] \times \Omega .
$$

The next result confirms that $g$ is a driver function (the proof is given in the Appendix). 
LEMMA 3.4. There exists a version of $g$ such that, for $\mathrm{dP} \times \mathrm{d} t$ a.e. $(t, \omega)$, $(h, \tilde{h}) \mapsto g(t, \omega, h, \tilde{h})$ is continuous, convex, positively-homogeneous and dominated by $g_{\lambda}$.

Note that $(t, \omega) \mapsto g(t, \omega, h, \tilde{h})$ is predictable for every $(h, \tilde{h}) \in \mathbb{R} \times L^{2}(v(\mathrm{~d} x))$ and by Lemma $3.4(h, \tilde{h}) \mapsto g(t, \omega, h, \tilde{h})$ is continuous in $(h, \tilde{h})$, so that by standard arguments $g$ can be approximated by $\mathcal{P} \otimes \mathcal{B}\left(\mathbb{R}^{d}\right) \otimes \mathcal{U}$-measurable step functions and $g$ itself may seen to be $\mathcal{P} \otimes \mathcal{B}\left(\mathbb{R}^{d}\right) \otimes \mathcal{U}$-measurable. Note further that $g(t, \omega, h, \tilde{h})$ is nonnegative as $R_{h, \tilde{h}}(t, \omega)$ is so for each $(h, \tilde{h})$, and $g(s, \omega, 0,0)=0$ since the density $R_{0,0}(s, \omega)$ of the measure $\mu_{0,0}$ is zero. In the next result, we show that $D_{0}$ may be identified with $D_{0}^{g}$.

LEMMA 3.5. Let $g$ be as in Lemma 3.4. For $X \in L^{2}\left(\mathcal{F}_{T}\right)$, we have $D_{0}(X)=$ $D_{0}^{g}(X)$.

Lemma 3.5 (which we prove in the Appendix) and Remark 1.2(ii) imply that $D_{t}=D_{t}^{g}$ not only for $t=0$ but also for all other $t \in(0, T]$. The proof is complete.

The proof of Lemma 3.3 is based on the following auxiliary result.

LEMMA 3.6. Let $D$ be a dynamic deviation measure and $t \in[0, T]$. If $A_{1}, \ldots, A_{n} \in \mathcal{F}_{t}$ and $A_{i} \cap A_{j}=\varnothing$ for $i \neq j$ and $X_{1}, \ldots, X_{n} \in L^{2}\left(\mathcal{F}_{T}\right)$, then for any $t \in[0, T]$,

$$
D_{t}\left(\sum_{i=1}^{n} I_{A_{i}} X_{i}\right)=\sum_{i=1}^{n} D_{t}\left(I_{A_{i}} X_{i}\right)
$$

ProOF. Set $S_{k}:=\sum_{i=1}^{k} I_{A_{i}} X_{i}$ and $B_{k}=\bigcup_{i=1}^{k} A_{i}, k=1, \ldots, n$. Let us first show by an induction argument that

$$
D_{t}\left(S_{n}\right)=\sum_{i=1}^{n} I_{A_{i}} D_{t}\left(X_{i}\right) .
$$

Equation (3.4) is a direct consequence of (3.5) and (1.1). Using (1.1) and the fact $B_{n-1} \cap A_{n}=\varnothing$, we have

$$
\begin{aligned}
D_{t}\left(S_{n}\right) & =D_{t}\left(I_{B_{n-1}} S_{n-1}+I_{B_{n-1}^{c}} I_{A_{n}} X_{n}\right)=I_{B_{n-1}} D_{t}\left(S_{n-1}\right)+I_{B_{n-1}^{c}} D_{t}\left(I_{A_{n}} X_{n}\right) \\
& =I_{B_{n-1}} \sum_{i=1}^{n-1} I_{A_{i}} D_{t}\left(X_{i}\right)+I_{B_{n-1}^{c}} I_{A_{n}} D_{t}\left(X_{n}\right)=\sum_{i=1}^{n} I_{A_{i}} D_{t}\left(X_{i}\right)
\end{aligned}
$$

where we used (1.1) and the induction assumption in the third equality. This completes the proof of (3.5) and hence of the lemma. 
3.2. $m$-Stability and time-consistency. We next turn to a dual representation result for general dynamic deviation measures which is, as we show in Theorem 3.12, given in terms of additively $m$-stable representing sets (see Definition 3.10). Specifically, we show that additive $m$-stability is in some sense necessary and sufficient to obtain the time-consistency axiom (D6); see Proposition 4.4. The proof of these results rests on auxiliary dual representation results. Using these results, we first establish in Theorem 3.7 that an integral representation of the form (2.5) holds for any dynamic deviation measure even if the domination condition is not satisfied.

In particular, we may strengthen the characterisation of dynamic deviation measures given in Proposition 3.2 as follows.

THEOREM 3.7. Let $D=\left(D_{t}\right)_{t \in[0, T]}$ be a collection of maps $D_{t}: L^{2}\left(\mathcal{F}_{T}\right) \rightarrow$ $L_{+}^{2}\left(\mathcal{F}_{t}\right), t \in[0, T]$. Then $D$ is a dynamic deviation measure if and only if there exists a convex positively homogeneous driver function $g$ such that for any $t \in$ $[0, T]$ and $X \in L^{2}\left(\mathcal{F}_{T}\right)$

$$
D_{t}(X)=\mathbb{E}\left[\int_{t}^{T} g\left(s, H_{s}^{X}, \tilde{H}_{s}^{X}\right) \mathrm{d} s \mid \mathcal{F}_{t}\right]
$$

Theorem 3.7 completely characterises DDMs in a continuous-time setting. It is remarkable that contrary to the characterization of nonlinear expectations of $g$-expectations [see, for instance, Coquet et al. (2002) or Hu et al. (2008)] a domination criterion for DDMs is not needed. The reason is as follows: For a dynamic deviation measure $D$, we can construct an increasing sequence of $g^{n}$, each of linear growth, with corresponding dynamic deviation measures $D^{g^{n}}$ such that

$$
\begin{aligned}
D(X) & =\lim _{n \rightarrow \infty} D^{g^{n}}(X)=\lim _{n \rightarrow \infty} \mathbb{E}\left[\int_{0}^{T} g^{n}\left(s, H_{s}^{X}, \tilde{H}_{s}^{X}\right) \mathrm{d} s\right] \\
& =\mathbb{E}\left[\int_{0}^{T} g\left(s, H_{s}, \tilde{H}_{s}\right) \mathrm{d} s\right],
\end{aligned}
$$

where $g:=\lim g^{n}$ and the last expectation is well defined by the monotone convergence theorem as $g^{n}$ is an increasing sequence and $D(X)$ is finite by assumption. For the characterization of nonlinear expectations through $g$-expectations even in a purely Brownian setting, however, we get

$$
\rho(X)=\lim _{n \rightarrow \infty} \rho^{n}(X)=\lim _{n \rightarrow \infty} \mathcal{E}^{g^{n}}(-X)=\lim _{n \rightarrow \infty} \mathbb{E}\left[\int_{0}^{T} g^{n}\left(s, Z_{s}^{n}\right) \mathrm{d} s\right] .
$$

We would like to conclude that the last term is equal to

$$
\mathbb{E}\left[\int_{0}^{T} g\left(s, Z_{s}\right) \mathrm{d} s\right] .
$$

But although we know that $g^{n}$ increases to a function $g$, we can in general not infer that $Z^{n}$ converges to a suitable process $Z$. If $g^{n}$ and $g$ would be Lipschitz, or 
of uniform linear or quadratic growth (which would be guaranteed by domination type criteria), there are results in the literature giving that the BSDE with driver $g$ has a solution $(Y, Z)$ and

$$
Z^{n} \stackrel{n \rightarrow \infty}{\longrightarrow} Z, \quad \mathcal{E}^{g^{n}}(-X) \stackrel{n \rightarrow \infty}{\longrightarrow} Y
$$

and, therefore, by (3.7) $\rho(X)=Y$. Hence, in this case the approach given above works also for nonlinear expectations. However, without such additional growth conditions on $g$ the convergence of $Z^{n}$ cannot be guaranteed and, therefore, also not the convergence of the last term in (3.7).

In the sequel, we give two examples where nonlinear expectations cannot be characterised by a $g$-expectation which in particular implies that the $Z^{n}$ in (3.7) will not converge to a well integrable limit (no matter actually how the approximation for the $g^{n}$ is chosen).

EXAMPLE 3.8 (No-arbitrage pricing). Suppose that we are in a classical incomplete financial market with two one-dimensional Brownian motions $\left(W^{1}, W^{2}\right)$ and a stock price process given by

$$
\mathrm{d} S_{t}=S_{t}\left(\left(\mathrm{~d} W_{t}^{1}+\mathrm{d} W_{t}^{2}\right)+\mu \mathrm{d} t\right)
$$

We assume that the interest rate of the bond is zero. The set of equivalent martingale measures is given by

$$
M:=\left\{\mathbb{Q} \ll \mathbb{P} \mid q_{s} \in C, s \in[0, T]\right\},
$$

where $\frac{\mathrm{d} \mathbb{Q}}{\mathrm{d} \mathbb{P}}=\mathcal{E}\left((q \cdot W)_{T}\right)$, with $\mathcal{E}$ denoting the stochastic exponential, and $C=$ $\left\{q=\left(\begin{array}{c}q_{1} \\ q_{2}\end{array}\right) \in \mathbb{R}^{2} \mid \mu+q_{1}+q_{2}=0\right\}$. Let us define $\rho_{t}: L^{\infty}\left(\mathcal{F}_{T}\right) \rightarrow L^{\infty}\left(\mathcal{F}_{t}\right)$ by

$$
\rho_{t}(X)=\underset{\mathbb{Q} \in M}{\operatorname{ess} \sup } \mathbb{E}_{\mathbb{Q}}\left[-X \mid \mathcal{F}_{t}\right]
$$

It is well known that $\rho$ is a time-consistent coherent risk measure; see Remark 3.13 below or Proposition 9.1 in Delbaen (2006). Define

$$
\mathcal{B}^{n}:=\left\{\left.\mathbb{Q} \ll \mathbb{P}|| q\right|^{2} \leq n^{2}\right\} \quad \text { and } \quad \rho_{t}^{n}(X):=\underset{\mathbb{Q} \in M \cap \mathcal{B}^{n}}{\operatorname{ess} \sup _{\mathbb{Q}}} \mathbb{E}_{\mathbb{Q}}\left[-X \mid \mathcal{F}_{t}\right]
$$

One may see that $\rho_{t}^{n}$ satisfies a domination criterion as the set $M \cap \mathcal{B}^{n}$ is weakly compact. In fact, $\rho^{n}$ may be related to good-deal bound pricing and one can prove [see Krätschmer et al. (2015)] that

$$
\rho_{t}^{n}(X)=\mathcal{E}^{g^{n}}(-X),
$$

where $\mathcal{E}^{g^{n}}$ is the $g$-expectation with driver function $g^{n}$ given by

$$
g^{n}(z)=\sqrt{n^{2}-\left|P_{B}(0)\right|^{2}}\left|P_{\operatorname{Kernel}(A)}(z)\right|+z P_{B}(0),
$$


where $A=(1,1), B=\left\{x \in \mathbb{R}^{2} \mid A x=-\mu\right\}$, and $P_{V}(x)$ denotes the projection of $x$ onto the space $V$. Hence,

$$
\rho_{t}(X)=\lim _{n \rightarrow \infty} \mathcal{E}^{g^{n}}(-X),
$$

but on $\left\{z \in \mathbb{R}^{2} \mid P_{\operatorname{Kernel}(A)}(z) \neq 0\right\}, g(z)=\lim _{n \rightarrow \infty} g^{n}(z)=\infty$. In particular, although $\rho_{t}$ is a time-consistent coherent risk measure, $\rho_{t}$ can not be characterised by a $g$-expectation.

EXAMPLE 3.9. The following example is inspired by Delbaen, $\mathrm{Hu}$ and Bao (2011). Let $\left(q_{t}^{*}\right)$ be a deterministic, positive process with

$$
\int_{0}^{T-\epsilon}\left|q_{t}^{*}\right|^{2} \mathrm{~d} t<\infty \quad \text { for every } \epsilon>0, \quad \text { and } \quad \int_{0}^{T}\left|q_{t}^{*}\right|^{2} \mathrm{~d} t=\infty .
$$

In particular, $\mathcal{E}\left(\left(q^{*} \cdot W\right)_{T}\right)=0$. Define further

$$
\sigma:=\inf \left\{t \in[0, T] \mid \mathcal{E}\left(\left(q^{*} \cdot W\right)_{t}\right) \geq n\right\},
$$

where $n$ is chosen large enough, so that $\mathbb{P}(\sigma=T)>0$. Define $\mathbb{Q}^{*}$ by

$$
\frac{\mathrm{d} \mathbb{Q}^{*}}{\mathrm{~d} \mathbb{P}}=\mathcal{E}\left(\left(q^{*} \cdot W\right)_{T \wedge \sigma}\right) .
$$

Note that $\mathbb{Q}^{*} \ll \mathbb{P}, \frac{\mathrm{d} \mathbb{Q}^{*}}{\mathrm{~d} \mathbb{P}}$ is bounded, $\mathbb{P}\left[\frac{\mathrm{d} \mathbb{Q}^{*}}{\mathrm{~d} \mathbb{P}}=0\right]>0$ and $\mathbb{Q}^{*} \sim \mathbb{P}$ on $\mathcal{F}_{t}$ for $t<T$.

Define $\rho_{t}: L^{2}\left(\mathcal{F}_{T}\right) \rightarrow L^{2}\left(\mathcal{F}_{t}\right)$ by $\rho_{T}(X):=-X$ and

$$
\rho_{t}(X):=\mathbb{E}_{\mathbb{Q}^{*}}\left[-X \mid \mathcal{F}_{t}\right] \quad \text { for } t \in[0, T) .
$$

Note that for $t<T$ we have that $\rho_{t}(X)$ is well defined a.s. as $\mathbb{Q}^{*} \sim \mathbb{P}$ on $\mathcal{F}_{t}$.

Clearly, $\rho_{t}$ is a coherent risk measure for every $t$. Furthermore, since we have $\rho_{t}\left(-\rho_{s}(X)\right)=\rho_{t}(X)$ for every $0 \leq t \leq s \leq T, \rho_{t}$ is also time-consistent. However, if we choose $\eta \in L_{+}^{\infty}\left(\mathcal{F}_{T}\right)$ with $\mathbb{P}[\eta>0]>0$ and $\mathbb{Q}^{*}[\eta>0]=0, \rho_{t}(\eta)$ cannot be the solution of a BSDE. This is seen as follows: As $\eta=0 \mathbb{Q}^{*}$-a.s., for $t<T$ we have

$$
\rho_{t}(\eta)=\rho_{t}(0)=0 \text {. }
$$

Now solutions of BSDEs are a.s. continuous in $t$. So, if $\left(\rho_{t}(\eta)\right)_{0 \leq t \leq T}$ was the solution of a BSDE, this would imply for $t^{n} \uparrow T$

$$
-\eta=\rho_{T}(\eta)=\lim _{n \rightarrow \infty} \rho_{t^{n}}(\eta)=0,
$$

which is a contradiction.

Note that in this counter-example the potential limiting BSDE corresponds to

$$
\begin{aligned}
Y_{T} & =-\eta, \\
\mathrm{d} Y_{t} & =-q_{t}^{*} Z_{t} \mathrm{~d} t+Z_{t} \mathrm{~d} W_{t}, \quad t \in[0, T) .
\end{aligned}
$$

This BSDE has a non-Lipschitz driver that explodes at $t=T$. 
The mentioned notion of additive $m$-stability is the requirement of stability under additive pasting of subsets of the collections of (conditionally) zero-mean random variables given by

$$
\mathcal{Q}_{\mathcal{F}_{t}}:=\left\{\xi \in L^{2}\left(\mathcal{F}_{T}\right) \mid \mathbb{E}\left[\xi \mid \mathcal{F}_{t}\right]=0\right\}, \quad \mathcal{Q}:=\mathcal{Q}_{\mathcal{F}_{0}}=\left\{\xi \in L^{2}\left(\mathcal{F}_{T}\right) \mid \mathbb{E}[\xi]=0\right\} .
$$

DEFINITION 3.10. A set $\mathcal{S} \subset \mathcal{Q}$ is called additively $m$-stable if for any $\xi^{1}, \xi^{2} \in \mathcal{S}$ and $t \in[0, T], \xi^{2}+\mathbb{E}\left[\xi^{1}-\xi^{2} \mid \mathcal{F}_{t}\right]$ defines an element of $\mathcal{S}$.

Denoting for a given set $\mathcal{S} \subset \mathcal{Q}$

$$
\mathcal{S}_{s, t}:=\left\{\mathbb{E}\left[\xi \mid \mathcal{F}_{t}\right]-\mathbb{E}\left[\xi \mid \mathcal{F}_{s}\right] \mid \xi \in \mathcal{S}\right\}, \quad s, t \in[0, T],
$$

we note that $\mathcal{S}=\mathcal{S}_{0, T}$ and that a necessary and sufficient condition for $\mathcal{S}$ to be additively $m$-stable is

$$
\mathcal{S}=\mathcal{S}_{0, t}+\mathcal{S}_{t, T} \quad \text { for any } t \in[0, T],
$$

where $A+B$ denotes the direct sum of the sets $A$ and $B .{ }^{6}$

THEOREM 3.11. Let $D=\left(D_{t}\right)_{t \in[0, T]}$ be a collection of maps $D_{t}: L^{2}\left(\mathcal{F}_{T}\right) \rightarrow$ $L^{0}\left(\mathcal{F}_{t}\right), t \in[0, T]$, satisfying (D4). Then $D$ is a dynamic deviation measure if and only if for some convex, bounded, closed subset $\mathcal{S}^{D}$ of $\mathcal{Q}$ that contains zero and is additively $m$-stable we have

$$
D_{t}(X)=\operatorname{esssup}_{\xi \in \mathcal{S}^{D} \cap \mathcal{Q}_{\mathcal{F}_{t}}} \mathbb{E}\left[\xi X \mid \mathcal{F}_{t}\right], \quad t \in[0, T]
$$

In the next result, we call a $\mathcal{P} \otimes \mathcal{B}\left(\mathbb{R}^{d}\right) \otimes \mathcal{U}$-measurable subset $C=\left(C_{t}\right)_{t \in[0, T]}$ of $[0, T] \times \Omega \times \mathbb{R}^{d} \times L^{2}(v(\mathrm{~d} x))$ closed, convex or nonempty if for $\mathrm{d} \mathbb{P} \times \mathrm{d} t$ a.e. $(t, \omega) \in[0, T] \times \Omega$, the sets $C_{t}(\omega)$ are closed, convex or nonempty. We denote by $\operatorname{int}(C)$ the collection of interiors of the sets $C_{t}(\omega),(t, \omega) \in[0, T] \times \Omega$.

THEOREM 3.12. Let $D=\left(D_{t}\right)_{t \in[0, T]}$ be a collection of maps $D_{t}: L^{2}\left(\mathcal{F}_{T}\right) \rightarrow$ $L^{0}\left(\mathcal{F}_{t}\right), t \in[0, T]$. Then $D$ is a dynamic deviation measure if and only if there exists a $\mathcal{P} \otimes \mathcal{B}\left(\mathbb{R}^{d}\right) \otimes \mathcal{U}$-measurable set $C^{D}=\left(C_{t}^{D}\right)_{t \in[0, T]}$ that is convex, closed with $0 \in \operatorname{int}\left(C^{D}\right)$, such that $D$ satisfies the representation in (3.8) with a bounded set $\mathcal{S}^{D}$ given in terms of $C^{D}$ by

$$
\mathcal{S}^{D}=\left\{\xi \in \mathcal{Q} \mid\left(H_{t}^{\xi}, \tilde{H}_{t}^{\xi}\right) \in C_{t}^{D} \text { for all } t \in[0, T]\right\}
$$

The proofs of Theorems 3.7, 3.11 and 3.12 are given below.

\footnotetext{
${ }^{6}$ That is, $A+B:=\{a+b: a \in A, b \in B\}$.
} 
REMARK 3.13 (Relation to strong time-consistency of dynamic risk-measures). The characterisation in Theorem 3.12 is reminiscent of analogous characterisation results of (strong) time-consistency of dynamic risk measures available in the literature. If we call a set $\mathcal{S}^{\prime} \subset \mathcal{M}$ multiplicatively $m$-stable if for every $\xi^{1}, \xi^{2} \in \mathcal{S}^{\prime}$ and $t \in[0, T]$ the element $L_{t}:=\xi_{t}^{1} \xi_{T}^{2} / \xi_{t}^{2}$ is contained in $\mathcal{S}^{\prime}$, we note that under multiplicative $m$-stability of $\mathcal{S}^{\prime}$ we have the decomposition $\mathcal{S}^{\prime}=\mathcal{S}_{0, T}^{\prime}=\mathcal{S}_{0, t}^{\prime} \mathcal{S}_{t, T}^{\prime}$ with $\mathcal{S}_{s, t}^{\prime}:=\left\{\mathbb{E}\left[\xi \mid \mathcal{F}_{t}\right] / \mathbb{E}\left[\xi \mid \mathcal{F}_{s}\right] \mid \xi \in \mathcal{S}^{\prime}\right\}$ (with $0 / 0=0$ ), so that the set $\mathcal{S}^{\prime}$ is stable under "multiplicative" pasting. It is well known that coherent risk measures are (strongly) time-consistent precisely if the representing sets in the corresponding dual representations are multiplicatively $m$-stable; see among many others Chen and Epstein (2002) (where multiplicative $m$-stablility is called "rectangular property"), Riedel (2004), Delbaen (2006), Artzner et al. (2007) or Föllmer and Schied (2011). Specifically, in a Brownian setting it is shown in Delbaen (2006) that multiplicative $m$-stability of a convex and closed set $\mathcal{S}^{\prime} \subset \mathcal{M}:=\left\{\xi \in L_{+}^{1}\left(\mathcal{F}_{T}\right) \mid \mathbb{E}[\xi]=1\right\}$ containing 1 corresponds to the existence of a $\mathcal{P} \otimes \mathcal{B}\left(\mathbb{R}^{d}\right) \otimes \mathcal{U}$-measurable, closed and convex set $C^{\prime}$ containing 0 such that $\mathcal{S}^{\prime}=\left\{\xi \in \mathcal{M} \mid\left(q_{s}^{\xi}, \psi_{s}^{\xi}\right) \in C_{s}^{\prime}\right.$ for all $\left.s \in[0, T]\right\}$, where $\left(q^{\xi}, \psi^{\xi}\right)$ is related to the stochastic logarithm of $\xi$ by $\xi=\mathcal{E}\left(\left(q^{\xi} \cdot W\right)_{T}+\left(\psi^{\xi} \cdot \tilde{N}\right)_{T}\right)$ with $\mathcal{E}(\cdot)$ denoting the Doléans-Dade exponential. This result implies that time-consistent coherent risk measures on $L^{\infty}$ satisfy the representation

$$
\rho_{t}(X)=\operatorname{ess}_{\xi \in \mathcal{S}^{\prime} \cap \mathcal{M}_{\mathcal{F}_{t}}} \mathbb{E}\left[-\xi X \mid \mathcal{F}_{t}\right]
$$

$$
\begin{gathered}
\text { with } \mathcal{M}_{\mathcal{F}_{t}}:=\left\{\xi \in L_{+}^{1}\left(\mathcal{F}_{T}\right) \mid \mathbb{E}\left[\xi \mid \mathcal{F}_{t}\right]=1\right\} \quad \text { and } \\
\mathcal{S}^{\prime}=\left\{\xi \in L_{+}^{1}\left(\mathcal{F}_{T}\right) \mid\left(q_{S}^{\xi}, \psi_{S}^{\xi}\right) \in C_{s}^{\prime} \text { for all } s \in[0, T]\right\} .
\end{gathered}
$$

This result is generalised in Delbaen et al. (2010) to convex risk measures. As a counterpart of Theorem 3.1 in Delbaen (2006), which concerns multiplicatively $m$-stable sets in a Brownian filtration, we have from Theorem 3.12 and Propositions 4.1-4.4 below that a closed and convex set $\mathcal{S} \subset \mathcal{Q}$ containing 0 is additively $m$-stable if and only if, for some $\mathcal{P} \otimes \mathcal{B}\left(\mathbb{R}^{d}\right) \otimes \mathcal{U}$-measurable set $C^{*}=\left(C_{t}^{*}\right)_{t \in[0, T]}$ that is convex, closed and contains 0 , we have $\mathcal{S}=\left\{\xi \in \mathcal{Q} \mid\left(H_{t}^{\xi}, \tilde{H}_{t}^{\xi}\right) \in C_{t}^{*}\right.$ for all $t \in[0, T]\}$.

While a static deviation measure can under additional assumptions be represented as a coherent risk measure plus an expectation, in a dynamic setting this is typically not possible. So a DDM under our assumptions, is not a conditional expectation plus a coherent risk measure, but a genuinely new stochastic process. In particular, our SDEs do not correspond to classical BSDEs (plus a conditional expectation) but constitute a different class of stochastic equations. In fact, the following proposition holds. 
Proposition 3.14. Let $D=D^{g}$ be a DDM with corresponding set $C^{D}=$ $\left(C_{t}^{D}\right)_{0 \leq t \leq T}$. Suppose that the boundary $\partial C_{t}^{D}(\omega)$ of the set $C_{t}^{D}(\omega)$ is deterministic in the sense that it does not depend on $\omega$. Define $\rho(X):=\mathbb{E}[-X]+\gamma D(X)$. Then $\rho$ satisfies translation invariance, positive homogeneity and subadditivity, but not monotonicity.

\section{Proofs.}

Auxiliary representation results. Our starting point is the $\mathcal{F}_{t}$-conditional version of the duality result given in Theorem 1 in Rockafellar, Uryasev and Zabarankin (2006a).

Proposition 4.1. Let $t \in[0, T]$ and let the map $D_{t}: L^{2}\left(\mathcal{F}_{T}\right) \rightarrow L^{0}\left(\mathcal{F}_{t}\right)$ be given:

(i) $D_{t}$ satisfies (D1)-(D3) and (D5) and maps $L^{2}\left(\mathcal{F}_{T}\right)$ to $L_{+}^{2}\left(\mathcal{F}_{t}\right)$ if and only if there exists a bounded, closed and convex set $\mathcal{S}_{D_{t}} \subset \mathcal{Q}_{\mathcal{F}_{t}}$ containing zero such that

$$
D_{t}(X)=\underset{\xi \in \mathcal{S}_{D_{t}}}{\operatorname{ess} \sup } \mathbb{E}\left[\xi X \mid \mathcal{F}_{t}\right], \quad X \in L^{2}\left(\mathcal{F}_{T}\right)
$$

The set $\mathcal{S}_{D_{t}}$ is uniquely determined by its (convex) indicator function $J_{\mathcal{S}_{D_{t}}}$ : $L^{2}\left(\mathcal{F}_{T}\right) \rightarrow\{0, \infty\}$ given by

$$
J_{\mathcal{S}_{D_{t}}}(\xi):=\operatorname{ess}_{X \in L^{2}\left(\mathcal{F}_{T}\right)}\left\{\mathbb{E}\left[\xi X \mid \mathcal{F}_{t}\right]-D_{t}(X)\right\} .
$$

(ii) Assume the conditions in (i) are satisfied. Then $D_{t}$ satisfies (D4) if and only if for every $X \in L^{2}\left(\mathcal{F}_{T}\right)$ with $X \notin L^{2}\left(\mathcal{F}_{t}\right)$ there exists $\xi \in \mathcal{S}_{D_{t}}$ such that $\mathbb{P}\left[\mathbb{E}\left[\xi X \mid \mathcal{F}_{t}\right]>0\right]>0$.

REMARK 4.2. Note that by (4.2) we have for any set $A \in \mathcal{F}_{t}$ and $\xi^{1}, \xi^{2} \in \mathcal{S}_{D_{t}}$ that $I_{A} \xi^{1}+I_{A^{c}} \xi^{2} \in \mathcal{S}_{D_{t}}$. Sets having this property are directed. ${ }^{7}$

Hence, $D_{t}(X)$ admits a robust representation with representing set given by a collection of signed measures. This proposition is stated in Rockafellar, Uryasev and Zabarankin (2006a) in a static setting but it can be seen to also hold true conditionally on $\mathcal{F}_{t}$; see, for instance, Riedel (2004), Ruszczyński and Shapiro (2006), or Cheridito and Kupper (2011) for related arguments.

For dynamic deviation measures the property, (D6) induces a specific structure of the sets $\mathcal{S}_{D_{t}}, t \in[0, T]$, which we specify in the next results. A first observation is as follows.

\footnotetext{
${ }^{7}$ A set $\mathcal{S}$ is called directed if for any $\xi^{1}, \xi^{2} \in \mathcal{S}$ there exists $\bar{\xi} \in \mathcal{S}$ with $\bar{\xi} \geq \xi^{1} \vee \xi^{2}$.
} 
Proposition 4.3. Let $t \in[0, T]$ and let $D$ be a dynamic deviation measure and denote $\mathcal{S}^{D}:=\mathcal{S}_{D_{T}}$. We have that the set $\mathcal{S}_{D_{t}}$ in the representation (4.1) of $D_{t}$ is such that $\mathcal{S}_{D_{t}}=\mathcal{S}^{D} \cap \mathcal{Q}_{\mathcal{F}_{t}}=\mathcal{S}_{t, T}^{D}$.

The following result shows that stability under "additive pasting" of the representing set in the form of additive $m$-stability is a necessary and sufficient condition for (D6) to hold.

Proposition 4.4. Let $\mathcal{S} \subset \mathcal{Q}$ be a convex, closed set containing zero. $\mathcal{S}$ is additively $m$-stable if and only if the collection $D_{t}(X):=$

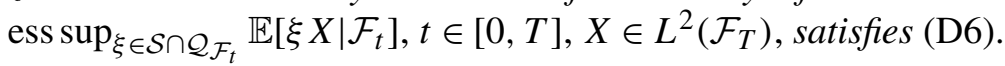

The proofs of Propositions 4.3 and 4.4 are given in the Appendix.

ProOF OF THEOREM 3.11. The assertion follows by combining Propositions 4.1, 4.3 and 4.4.

In the proofs of Theorems 3.7 and 3.12, we deploy, for a given dynamic deviation measure $D$, the sequence $\left(D^{(n)}\right)_{n \in \mathbb{N}}$ of dynamic deviation measures $D^{(n)}=\left(D_{t}^{(n)}\right)_{t \in[0, T]}, D_{t}^{(n)}: L^{2}\left(\mathcal{F}_{T}\right) \rightarrow L^{2}\left(\mathcal{F}_{t}\right)$ defined by

$$
\begin{aligned}
D_{t}^{(n)}(X) & :=\underset{\xi \in\left(\mathcal{S}^{D} \cap \mathcal{Q}_{\mathcal{F}_{t}}\right) \cap \mathcal{A}^{n}}{\operatorname{ess} \sup } \mathbb{E}\left[\xi X \mid \mathcal{F}_{t}\right] \quad \text { with } \\
\mathcal{A}^{n} & :=\left\{\xi \in L^{2}\left(\mathcal{F}_{T}\right) \mid \sup _{s \in[0, T]}\left\{\left|H_{s}^{\xi}\right|^{2}+\int_{\mathbb{R}^{k} \backslash\{0\}}\left|\tilde{H}_{s}^{\xi}(x)\right|^{2} v(\mathrm{~d} x)\right\} \leq n^{2}\right\} .
\end{aligned}
$$

LEMMA 4.5. Let $t \in[0, T]$ and $X \in L^{2}\left(\mathcal{F}_{T}\right)$ and, for a given dynamic deviation measure D, let $\left(D^{(n)}\right)_{n \in \mathbb{N}}$ and $\left(\mathcal{A}^{n}\right)_{n \in \mathbb{N}}$ be as in (4.3)-(4.4).

(i) For any $n \in \mathbb{N}$, we have $D_{t}^{(n)}(X) \leq D_{t}^{(n+1)}(X)$ and $\mathcal{A}^{n+1}=\frac{n+1}{n} \mathcal{A}^{n}$. Moreover, $D_{t}^{(n)}(X) \nearrow D_{t}(X)$ in $L^{2}\left(\mathcal{F}_{t}\right)$ as $n \rightarrow \infty$.

(ii) For any $n \in \mathbb{N}, \mathcal{S} \cap \mathcal{A}^{n}$ contains zero and is closed, bounded, convex and additively $m$-stable.

(iii) For any $n \in \mathbb{N}, D^{(n)}$ is a dynamic deviation measure that is $n$-dominated.

PROOF. (i) It is easily verified that $\mathcal{A}^{n+1}=\frac{n+1}{n} \mathcal{A}^{n}$ so that $\mathcal{A}^{n} \subset \mathcal{A}^{n+1}$ for $n \in$ $\mathbb{N}$. Hence, by (4.3) we have $D_{t}^{(n)}(X) \leq D_{t}^{(n+1)}(X)$ for $t \in[0, T]$ and $X \in L^{2}\left(\mathcal{F}_{T}\right)$. Furthermore, as $\left(\mathcal{A}^{n}\right)_{n \in \mathbb{N}}$ is dense in $L^{2}\left(\mathcal{F}_{T}\right)$ and the set $\mathcal{S}^{D}$ in Theorem 3.11 is bounded, we have that $D_{t}^{(n)}(X) \nearrow D_{t}(X)$ as $n \rightarrow \infty$.

(ii) Let $n \in \mathbb{N}$. It is straightforward to verify that $\mathcal{A}^{n}$ contains zero and is closed, bounded and convex. Let us show next that $\mathcal{A}^{n}$ is additively $m$-stable. Let $t \in[0, T]$ and $\xi^{1}, \xi^{2} \in \mathcal{A}^{n}$ and denote $L=\xi^{2}+\mathbb{E}\left[\xi^{1}-\xi^{2} \mid \mathcal{F}_{t}\right]$. Then the representing pair 
$\left(H^{L}, \tilde{H}^{L}\right)$ of $L \in L^{2}\left(\mathcal{F}_{T}\right)$ is expressed in terms of the representing pairs $\left(H^{i}, \tilde{H}^{i}\right)$, $i=1,2$, of $\xi^{1}, \xi^{2}$ by $H_{s}^{L}=H_{s}^{1} I_{[0, t]}(s)+H_{s}^{2} I_{(t, T]}(s)$ and $\tilde{H}_{s}^{L}=\tilde{H}_{s}^{1} I_{[0, t]}(s)+$ $\tilde{H}_{S}^{2} I_{(t, T]}(s)$. In particular, we have $\sup _{s \in[0, T]}\left\{\left|H_{s}^{L}\right|^{2}+\int_{\mathbb{R}^{k} \backslash\{0\}}\left|\tilde{H}_{s}^{L}(x)\right|^{2} v(\mathrm{~d} x)\right\} \leq$ $n^{2}$ so that $L \in \mathcal{A}^{n}$. Thus, $\mathcal{A}^{n}$ is additively $m$-stable. Since the set $\mathcal{S}^{D}$ is also closed, convex and additively $m$-stable, the same holds for $\mathcal{A}^{n} \cap \mathcal{S}$.

(iii) Let $n \in \mathbb{N}$. From Proposition 4.1 and part (ii), we conclude that $D^{(n)}$ satisfies (D1)-(D3) and (D5). Furthermore, from Proposition 4.4 and part (ii) we have that $D^{(n)}$ satisfies (D6). Let us show next that $D^{(n)}$ satisfies positivity (D4). Let $t \in[0, T]$ and $X \in L^{2}\left(\mathcal{F}_{T}\right) \backslash L^{2}\left(\mathcal{F}_{t}\right)$. By Propositions 4.1 and 4.3, there exists a $\tilde{\xi} \in \mathcal{S}^{D} \cap \mathcal{Q}_{\mathcal{F}_{t}}$ such that $\mathbb{E}\left[\tilde{\xi} X \mid \mathcal{F}_{t}\right]>0$ on a nonzero set. As $\left(\mathcal{A}^{n}\right)_{n \in \mathbb{N}}$ is increasing and dense in $L^{2}\left(\mathcal{F}_{T}\right)$ [as noted in the proof of part (i)], we can find a sequence $\left(\xi^{m}\right)_{m}$ such that $\xi^{m} \in \mathcal{S}^{D} \cap \mathcal{Q}_{\mathcal{F}_{t}} \cap \mathcal{A}^{m}$ converges to $\tilde{\xi}$ in $L^{2}\left(\mathcal{F}_{T}\right)$ as $m \rightarrow \infty$. Next, choose $m^{\prime}$ sufficiently large such that on a nonzero set, say $A$, we have $\mathbb{E}\left[\xi^{m^{\prime}} X \mid \mathcal{F}_{t}\right]>0$ (which is possible since $\xi^{m} X$ converges to $\tilde{\xi} X$ in $L^{1}$ as $m \rightarrow \infty)$. Define $\xi^{*} \in \mathcal{S}^{D} \cap \mathcal{Q}_{\mathcal{F}_{t}} \cap \mathcal{A}^{n}$ by $\xi^{*}:=\frac{n}{m^{\prime}} \xi^{m^{\prime}}$. Since on $A$ we have $\mathbb{E}\left[\xi^{*} X \mid \mathcal{F}_{t}\right]=\frac{n}{m^{\prime}} \mathbb{E}\left[\tilde{\xi}^{m^{\prime}} X \mid \mathcal{F}_{t}\right]>0$ we conclude from (4.3) that $D^{(n)}$ satisfies (D4).

Finally, by deploying the Cauchy-Schwarz inequality we note that $D_{t}^{(n)}(X)$ may be bounded above by

$$
\begin{aligned}
\sup _{\xi \in \mathcal{A}^{n}} \mathbb{E}\left[\xi X \mid \mathcal{F}_{t}\right] & =\sup _{\xi \in \mathcal{A}^{n}} \mathbb{E}\left[\int_{0}^{T}\left(\left(H_{s}^{\xi}\right)^{\top} H_{s}^{X}+\int_{\mathbb{R}^{k} \backslash\{0\}} \tilde{H}_{s}^{\xi}(x) \tilde{H}_{s}^{X}(x) v(\mathrm{~d} x)\right) \mathrm{d} s \mid \mathcal{F}_{t}\right] \\
& \leq n \mathbb{E}\left[\int_{0}^{T} \sqrt{\left|H_{s}^{X}\right|^{2}+\int_{\mathbb{R}^{k} \backslash\{0\}}\left|\tilde{H}_{s}^{X}(x)\right|^{2} v(\mathrm{~d} x)} \mathrm{d} s \mid \mathcal{F}_{t}\right] \\
& =\bar{D}_{t}^{n}(X),
\end{aligned}
$$

where we denote by $v^{\top}$ the transpose of the column vector $v \in \mathbb{R}^{d}$.

4.1. Proof of Theorem 3.7. With the previously established results in hand, we can now complete the proof of Theorem 3.7. As the arguments in the proof of the implication " $\Leftarrow$ " in Proposition 3.2 carry over for the proof of " $\Leftarrow$ " in Theorem 3.7, the remainder of the proof is concerned with the proof of " $\Rightarrow$ ". Let $D$ be a dynamic deviation measure, $X \in L^{2}\left(\mathcal{F}_{T}\right)$ and denote by $\left(D^{(n)}\right)_{n \in \mathbb{N}}$ the approximating sequence of dynamic deviation measures from Lemma 4.5. By Lemma 4.5(i), (iii) and Proposition 3.2 the sequence $\left(D^{(n)}(X)\right)_{n \in \mathbb{N}}$ is monotone increasing and there exists a sequence $\left(g^{n}\right)_{n \in \mathbb{N}}$ of convex and positively homogeneous driver functions such that (3.6) holds (with $D$ and $g$ replaced by $D^{(n)}$ and $g^{n}$ ). Therefore, by Proposition 2.6(iv), $g^{n} \leq g^{n+1}$ for $n \in \mathbb{N}$, so that we can define $g:=\lim _{n \rightarrow \infty} g^{n}$. Clearly, $g$ is convex, positively homogeneous and lower semcontinuous as the limit of functions having these properties. Furthermore, for $(h, \tilde{h}) \neq 0$ we have $g(\omega, t, h, \tilde{h}) \geq g^{1}(\omega, t, h, \tilde{h})>0$ and $g(\omega, t, 0,0)=$ 
$\lim _{n \rightarrow \infty} g^{n}(\omega, t, 0,0)=0 \mathrm{~d} \mathbb{P} \times \mathrm{d} t$ a.e. Hence, $g$ is a convex and positively homogeneous driver function. Finally, as $\left(g^{n}\right)_{n}$ is an increasing sequence of functions an application of the monotone convergence theorem yields

$$
\begin{aligned}
D_{t}(X) & =\lim _{n} D_{t}^{(n)}(X)=\lim _{n} \mathbb{E}\left[\int_{t}^{T} g^{n}\left(s, H_{s}^{X}, \tilde{H}_{s}^{X}\right) \mathrm{d} s \mid \mathcal{F}_{t}\right] \\
& =\mathbb{E}\left[\int_{t}^{T} g\left(s, H_{s}^{X}, \tilde{H}_{s}^{X}\right) \mathrm{d} s \mid \mathcal{F}_{t}\right] .
\end{aligned}
$$

This completes the proof of Theorem 3.7.

4.2. Proof of Theorem 3.12. In the proof of Theorem 3.12, we deploy the following auxiliary result.

LEMMA 4.6. (i) Let $g$ be a convex and positively homogeneous driver function and let the $\mathcal{P} \otimes \mathcal{B}\left(\mathbb{R}^{d}\right) \otimes \mathcal{U}$-measurable set $C=\left(C_{t}\right)_{t \in[0, T]}$ be determined by

$$
\begin{aligned}
J_{C_{t}}(u, \tilde{u}) & =r(t, u, \tilde{u}) \\
& :=\sup _{u \in \mathbb{R}^{d}, \tilde{u} \in L^{2}(v(\mathrm{~d} x))}\left\{u^{\top} h+\int_{\mathbb{R}^{k} \backslash\{0\}} \tilde{u}(x) \tilde{h}(x) v(\mathrm{~d} x)-g(t, u, \tilde{u})\right\}
\end{aligned}
$$

for $u \in \mathbb{R}^{d}$ and $\tilde{u} \in L^{2}(v(\mathrm{~d} x))$. Then $0 \in \operatorname{int}\left(C_{t}\right)(\omega) \mathrm{d} \mathbb{P} \times \mathrm{d} t$ a.e.

(ii) Let $C^{D}=\left(C_{t}^{D}\right)_{t \in[0, T]}$ be a $\mathcal{P} \otimes \mathcal{B}\left(\mathbb{R}^{d}\right) \otimes \mathcal{U}$-measurable set and let $\mathcal{S}^{D}$ be given by the right-hand side of (3.9). If $0 \in \operatorname{int}\left(C_{t}^{D}\right)(\omega) \mathrm{d} \mathbb{P} \times \mathrm{d} t$ a.e. then, for any $t \in[0, T]$ and $X \in L^{2}\left(\mathcal{F}_{T}\right) \backslash L^{2}\left(\mathcal{F}_{t}\right)$, there exists a $\xi^{\prime} \in \mathcal{S}^{D}$ such that $\mathbb{P}\left(\mathbb{E}\left[\xi^{\prime} X \mid \mathcal{F}_{t}\right]>0\right)>0$.

Proof. To simplify notation, we denote $z:=(h, \tilde{h})$ and $y=(q, \psi)$ for elements $(h, \tilde{h}),(q, \psi)$ in the Hilbert space $\mathbb{R}^{d} \times L^{2}(v(\mathrm{~d} x))$. Further, we denote $\langle y, z\rangle_{*}=q h+\int_{\mathbb{R}^{k} \backslash\{0\}} \psi(x) \tilde{h}(x) v(\mathrm{~d} x)$ and $|z|_{*}=\sqrt{|h|^{2}+\int_{\mathbb{R}^{k} \backslash\{0\}}|\tilde{h}(x)|^{2} v(\mathrm{~d} x)}$.

(i) Set $\mathcal{Z}:=\left\{z \in \mathbb{R}^{d} \times L^{2}(v(\mathrm{~d} x)) \|\left. z\right|_{*}=1\right\}$ and for $z \in \mathcal{Z}$ and $\lambda \in \mathbb{R}$ we denote $z^{\lambda}:=\lambda z$. By the positive homogeneity of $g$ and the symmetry of the set $\mathcal{Z}$, we have for fixed $y \in \mathbb{R}^{d} \times L^{2}(v(\mathrm{~d} x))$ that $r(t, y)=\sup _{z \in \mathcal{Z}, \lambda \in \mathbb{R}}\left\{\left\langle y, z^{\lambda}\right\rangle_{*}-g\left(t, z^{\lambda}\right)\right\}$ is equal to

$$
\text { (4.6) } r(t, y)=\sup _{z \in \mathcal{Z}, \lambda \geq 0}\left\{\left\langle y, z^{\lambda}\right\rangle_{*}-g\left(t, z^{\lambda}\right)\right\}=\sup _{z \in \mathcal{Z}, \lambda \geq 0} \lambda\left\{\langle y, z\rangle_{*}-g(t, z)\right\} \text {. }
$$

The supremum in (4.6) is finite (and in this case equal to zero) if and only if for all $z \in \mathcal{Z}\langle y, z\rangle_{*} \leq g(t, z)$. Letting $\left(y_{n}\right)_{n}$ be a sequence such that $\left|y_{n}\right|_{*} \rightarrow 0$ and using the Cauchy-Schwarz inequality, we have that

$$
\sup _{z \in \mathcal{Z}}\left|\left\langle y_{n}, z\right\rangle_{*}\right| \leq\left|y_{n}\right|_{*} \sup _{z \in \mathcal{Z}}|z|_{*}=\left|y_{n}\right|_{*} \rightarrow 0 .
$$


Since by assumption $g(t, z)>0$ for every $z \in \mathcal{Z}$ it follows then from (4.6) that from a certain $n$ onwards $r\left(t, y_{n}\right)=0$. As $r\left(t, \omega, y_{n}\right)=J_{C_{t}(\omega)}\left(y_{n}\right)$ this entails that $y_{n} \in C_{t}(\omega)$ from a certain $n$ onwards for every sequence $y_{n}$ that is such that $\left|y_{n}\right|_{*} \rightarrow 0$. Hence, $0 \in \operatorname{int}\left(C_{t}(\omega)\right)$.

(ii) Let $t \in[0, T]$ and $X \in L^{2}\left(\mathcal{F}_{T}\right) \backslash L^{2}\left(\mathcal{F}_{t}\right)$. For any $s \in[0, T]$, we note that if $0 \in \operatorname{int}\left(C_{s}^{D}(\omega)\right)$ then there exists $\varepsilon_{s}^{\prime}(\omega) \in(0,1]$ such that $|y|_{*} \leq \varepsilon_{s}^{\prime}(\omega)$ implies $y \in C_{s}^{D}(\omega)$. Define $\lambda_{s}(\omega):=\left|\left(H_{s}^{X}(\omega), \tilde{H}_{s}^{X}(\omega)\right)\right|_{*}^{2}, A=\{(s, \omega) \in[t, T] \times$ $\left.\Omega: \lambda_{S}(\omega)>0\right\}$ and denote by $\varepsilon=\left(\varepsilon_{S}\right)_{s \in[0, T]}$ the process given by $\varepsilon_{s}(\omega):=$ $I_{A}(s, \omega) \varepsilon_{s}^{\prime}(\omega) / \lambda_{s}(\omega)$. Then $\xi^{\prime}:=\left(\varepsilon H^{X} \cdot W\right)_{t, T}+\left(\varepsilon \tilde{H}^{X} \cdot \tilde{N}\right)_{t, T}$ is element of $\mathcal{S}^{D}$. Since $X \in L^{2}\left(\mathcal{F}_{T}\right) \backslash L^{2}\left(\mathcal{F}_{t}\right)$, the set $A$ has positive $\mathrm{d} \mathbb{P} \times \mathrm{d} t$-measure so that

$$
\mathbb{E}\left[\mathbb{E}\left[X \xi^{\prime} \mid \mathcal{F}_{t}\right]\right]=\mathbb{E}\left[\mathbb{E}\left[\int_{t}^{T} I_{A} \varepsilon_{s}^{\prime} \mathrm{d} s \mid \mathcal{F}_{t}\right]\right]=\mathbb{E}\left[\int_{t}^{T} I_{A} \varepsilon_{s}^{\prime} \mathrm{d} s\right]>0,
$$

which implies, as $\mathbb{E}\left[X \xi^{\prime} \mid \mathcal{F}_{t}\right]$ is nonnegative, that $\mathbb{P}\left(\mathbb{E}\left[X \xi^{\prime} \mid \mathcal{F}_{t}\right]>0\right)>0$.

ProOF OF THEOREM 3.12. Let us first show the implication " $\Leftarrow$ ": We note first that, as is straightforward to verify, $\mathcal{S}^{D}$ given in (3.9) is additively $m$-stable, convex, bounded, closed and contains zero. Moreover, Lemma 4.6 and Proposition 4.1(ii) imply that, for any $t \in[0, T], D_{t}: L^{2}\left(\mathcal{F}_{T}\right) \rightarrow L^{2}\left(\mathcal{F}_{t}\right)$ defined by (3.8) satisfies (D4). Hence, by Theorem $3.11 D=\left(D_{t}\right)_{t \in[0, T]}$ is a dynamic deviation measure.

We next turn to the proof of " $\Rightarrow$ ". In view of Theorem 3.11 it suffices to show that $\mathcal{S}^{D}$ is given by the expression in (3.9). For any $n \in \mathbb{N}$, let $D^{(n)}$ be defined as in (4.3). As noted before $\left(D^{(n)}\right)_{n \in \mathbb{N}}$ is a collection of dynamic deviation measures increasing to $D$ (Lemma 4.5) and the corresponding sequence $\left(g^{n}\right)_{n \in \mathbb{N}}$ of driver functions is increasing and satisfies $g^{n} \leq g$ [Proposition 2.6(iv)], where $g$ is the function in the representation (3.6) of $D$ (in Theorem 3.7). For $u \in \mathbb{R}^{d}$, $\tilde{u} \in L^{2}(v(\mathrm{~d} x))$ and $n \in \mathbb{N}$ define

$$
r^{n}(s, u, \tilde{u}):=\sup _{h \in \mathbb{Q}^{d}, \tilde{h} \in\left\{h_{1}, h_{2}, h_{3}, \ldots\right\}}\left\{u^{\top} h+\int_{\mathbb{R}^{k} \backslash\{0\}} \tilde{u}(x) \tilde{h}(x) v(\mathrm{~d} x)-g^{n}(s, h, \tilde{h})\right\},
$$

where $\left\{h_{1}, h_{2}, h_{3}, \ldots\right\}$ denotes a countable basis of $L^{2}(v(\mathrm{~d} x))$. Note that for any $n \in \mathbb{N}$ we have (i) $r^{n}$ lower semicontinuous and convex in $(u, \tilde{u})$ and (ii) $r^{n}$ is a (convex) indicator function of some convex and closed set, say $C^{n}=\left(C_{s}^{n}\right)_{s \in[0, T]}$. Furthermore, we note the following observations: (a) since $r^{n}$ is the supremum of a $\mathcal{P} \otimes \mathcal{B}\left(\mathbb{R}^{d}\right) \otimes \mathcal{U}$ measurable process and $C^{n}$ is the set where $r^{n}$ is equal to zero, we have that $C^{n}$ is also $\mathcal{P} \otimes \mathcal{B}\left(\mathbb{R}^{d}\right) \otimes \mathcal{U}$-measurable and (b) as the functions $g^{n}(s, h, \tilde{h})$ is continuous in $(h, \tilde{h}), r^{n}$ coincides with the dual conjugate of $g^{n}$, so that we have $\mathrm{d} \mathbb{P} \times \mathrm{d} t$ a.e.

$$
g^{n}(s, \omega, h, \tilde{h})=\sup _{(u, \tilde{u}) \in C_{s}^{n}(\omega)}\left\{u^{\top} h+\int_{\mathbb{R}^{k} \backslash\{0\}} \tilde{u}(x) \tilde{h}(x) v(\mathrm{~d} x)\right\} .
$$


Moreover, we have that (c) as the sequence $\left(g^{n}\right)_{n}$ is increasing, $\left(r^{n}\right)_{n}$ is a decreasing sequence so that $C^{n} \subset C^{n+1}$ for any $n \in \mathbb{N}$. Denote $C=\bigcup_{n=1}^{\infty} C^{n}$ and note that $C$ is convex and measurable as the increasing union of convex and measurable sets.

Let us next establish the representation (3.8) for $D^{(n)}(X)$ for given $n \in \mathbb{N}$ and $X \in L^{2}\left(\mathcal{F}_{T}\right)$. As $D^{(n)}(X)=D^{g^{n}}(X)$, we have

$$
\begin{aligned}
D_{0}^{(n)}(X)= & \mathbb{E}\left[\int_{0}^{T} \sup _{(u, \tilde{u}) \in C_{s}^{n}}\left(u^{\top} H_{s}^{X}+\int_{\mathbb{R}^{k} \backslash\{0\}} \tilde{u}(x) \tilde{H}_{s}^{X}(x) v(\mathrm{~d} x)\right) \mathrm{d} s\right] \\
\geq & \sup _{\left\{(H, \tilde{H}) \mid\left(H_{s}, \tilde{H}_{s}\right) \in C_{s}^{n}, s \in[0, T]\right\}} \mathbb{E}\left[\int _ { 0 } ^ { T } \left(H_{s}^{\top} H_{s}^{X}\right.\right. \\
& \left.\left.+\int_{\mathbb{R}^{k} \backslash\{0\}} \tilde{H}_{s}(x) \tilde{H}_{s}^{X}(x) v(\mathrm{~d} x)\right) \mathrm{d} s\right] \\
= & \sup _{\xi \in \mathcal{M}^{n}} \mathbb{E}\left[\int_{0}^{T}\left(\left(H_{s}^{\xi}\right)^{\top} H_{s}^{X}+\int_{\mathbb{R}^{k} \backslash\{0\}} \tilde{H}_{s}^{\xi}(x) \tilde{H}_{s}^{X}(x) v(\mathrm{~d} x)\right) \mathrm{d} s\right],
\end{aligned}
$$

with $\mathcal{M}^{n}:=\left\{\xi \in \mathcal{Q} \mid\left(H_{s}^{\xi}, \tilde{H}_{s}^{\xi}\right) \in C_{s}^{k}, s \in[0, T]\right\}$, where the supremum in (4.8) is taken over pairs $(H, \tilde{H}) \in L_{d}^{2}(\mathcal{P}, \mathrm{d} \mathbb{P} \times \mathrm{d} t) \times L^{2}\left(\mathcal{P} \times \mathcal{B}\left(\mathbb{R}^{k} \backslash\{0\}\right), \mathrm{d} \mathbb{P} \times \mathrm{d} t \times v(\mathrm{~d} x)\right)$.

Let us show next that the inequality in (4.8) is in fact an equality. It is well known [see, for instance, Theorem 2.4.9 in Zălinescu (2002)] that the subgradients of continuous and convex functions are nonempty so that the suprema in the dual representations of the functions $g^{n}, n \in \mathbb{N}$, are attained. Hence, we can apply a measurable selection theorem to the set

$$
\begin{aligned}
G^{n}:= & \left\{(s, \omega, u, \tilde{u}) \mid g^{n}\left(s, \omega, H_{s}^{X}, \tilde{H}_{s}^{X}\right)-u^{\top} H_{s}^{X}\right. \\
& \left.-\int_{\mathbb{R}^{k} \backslash\{0\}} \tilde{u}(x) \tilde{H}_{s}^{X}(x) v(\mathrm{~d} x)+J_{C_{s}^{n}(\omega)}(u, \tilde{u})=0\right\},
\end{aligned}
$$

obtaining $\mathcal{P} \times \mathcal{P} \otimes \mathcal{U}$-measurable processes $\left(U^{n}, \tilde{U}^{n}\right)$ such that, for every $s$, $\left(U_{s}^{n}, \tilde{U}_{s}^{n}\right) \in C_{s}^{n}$ and $g^{n}\left(s, H_{s}^{X}, \tilde{H}_{s}^{X}\right)=\left(U_{s}^{n}\right)^{\top} H_{s}^{X}+\int_{\mathbb{R}^{k} \backslash\{0\}} \tilde{U}_{s}^{n}(x) \tilde{H}_{s}^{X}(x) v(\mathrm{~d} x)$. This implies (4.8) holds with equality, and yields the desired representation for $D^{(n)}$.

To see that we also get a representation for $D$, let us first prove that the set $C$ defined above [our natural candidate to satisfy (3.8)-(3.9)] is closed. Note that from (4.9) it follows that for any $X \in L^{2}\left(\mathcal{F}_{T}\right)$

$$
\begin{aligned}
\sup _{\xi \in \mathcal{S} \cap \mathcal{A}^{n}} \mathbb{E}[\xi X] & =D_{0}^{(n)}(X) \\
& =\sup _{\xi \in \mathcal{M}^{n}} \mathbb{E}\left[\int_{0}^{T}\left(\left(H_{S}^{\xi}\right)^{\top} H_{s}^{X}+\int_{\mathbb{R}^{k} \backslash\{0\}} \tilde{H}_{S}^{\xi}(x) \tilde{H}_{s}^{X}(x) v(\mathrm{~d} x)\right) \mathrm{d} s\right] .
\end{aligned}
$$


As $\mathcal{S} \cap \mathcal{A}^{n}$ and $\mathcal{M}^{n}$ are both convex and closed sets, we conclude from (4.10) $\mathcal{S} \cap$ $\mathcal{A}^{n}=\mathcal{M}^{n}$. In particular, for $m \geq n$ we have $\mathcal{M}^{n}=\mathcal{M}^{m} \cap \mathcal{A}^{n}$. As there is a oneto-one correspondence between $\xi \in \mathcal{Q}$ and square-integrable predictable processes $(H, \tilde{H})$ this entails that

$$
\begin{aligned}
C^{n}= & C^{m} \cap\left\{(H, \tilde{H}) \in L^{2}(\mathrm{~d} \mathbb{P} \times \mathrm{d} t)\right. \\
& \left.\times L^{2}(\mathrm{~d} \mathbb{P} \times \mathrm{d} t \times v(\mathrm{~d} x)) \mid \sup _{t \in[0, T]}\left\{\left|H_{t}\right|^{2}+\int_{\mathbb{R}^{k} \backslash\{0\}}\left|\tilde{H}_{t}(x)\right|^{2} v(\mathrm{~d} x)\right\} \leq n^{2}\right\} .
\end{aligned}
$$

Hence, $\mathrm{d} \mathbb{P} \times \mathrm{d} t$ a.e.

$$
C_{t}^{n}(\omega)=C_{t}^{m}(\omega) \cap\left\{(h, \tilde{h}) \in \mathbb{R}^{d} \times\left. L^{2}(v(\mathrm{~d} x))|| h\right|^{2}+\int_{\mathbb{R}^{k} \backslash\{0\}}|\tilde{h}(x)|^{2} v(\mathrm{~d} x) \leq n^{2}\right\} .
$$

Taking the union over all $m \in \mathbb{N}$ on the right-hand side of previous display yields

$$
C_{t}^{n}(\omega)=C_{t}(\omega) \cap\left\{(h, \tilde{h}) \in \mathbb{R}^{d} \times\left. L^{2}(v(\mathrm{~d} x))|| h\right|^{2}+\int_{\mathbb{R}^{k} \backslash\{0\}}|\tilde{h}(x)|^{2} v(\mathrm{~d} x) \leq n^{2}\right\} .
$$

Since the sets $C_{t}^{n}(\omega), n \in \mathbb{N}$, are closed in $\mathbb{R}^{d} \times L^{2}(v(\mathrm{~d} x))$, we have that also $C_{t}(\omega)$ is closed.

As $g^{n}, n \in \mathbb{N}$, are convex positively homogeneous driver functions it follows by Lemma 4.6 that $0 \in \operatorname{int}\left(C^{n}\right)$. As $C^{n} \subset C$, we have thus that $0 \in \operatorname{int}(C)$.

Finally, to show that $C$ satisfies the desired representation (3.8)-(3.9) we note that $D_{0}(X)$ is equal to

$$
\begin{aligned}
\sup _{n \in \mathbb{N}} D_{0}^{(n)}(X)= & \sup _{n \in \mathbb{N}} \sup _{\left\{(H, \tilde{H}) \mid\left(H_{s}, \tilde{H}_{s}\right) \in C_{s}^{n}, s \in[0, T]\right\}} \mathbb{E}\left[\int _ { 0 } ^ { T } \left(H_{s}^{\top} H_{s}^{X}\right.\right. \\
& \left.\left.+\int_{\mathbb{R}^{k} \backslash\{0\}} \tilde{H}_{s}(x) \tilde{H}_{s}^{X}(x)\right) v(\mathrm{~d} x) \mathrm{d} s\right] \\
= & \sup _{\left\{(H, \tilde{H}) \mid\left(H_{s}, \tilde{H}_{s}\right) \in C_{s}, s \in[0, T]\right\}} \mathbb{E}\left[\int _ { 0 } ^ { T } \left(H_{s}^{\top} H_{s}^{X}\right.\right. \\
& \left.\left.+\int_{\mathbb{R}^{k} \backslash\{0\}} \tilde{H}_{s}(x) \tilde{H}_{s}^{X}(x) v(\mathrm{~d} x)\right) \mathrm{d} s\right] \\
= & \sup _{\left\{\xi \in \mathcal{Q} \mid\left(H_{s}^{\xi}, \tilde{H}_{s}^{\xi}\right) \in C_{s}, s \in[0, T]\right\}}^{\mathbb{E}[\xi X],}
\end{aligned}
$$

where in the first and second line the suprema are taken over pairs $(H, \tilde{H}) \in$ $L_{d}^{2}(\mathcal{P}, \mathrm{d} \mathbb{P} \times \mathrm{d} t) \times L^{2}\left(\mathcal{P} \times \mathcal{B}\left(\mathbb{R}^{k} \backslash\{0\}\right), \mathrm{d} \mathbb{P} \times \mathrm{d} t \times v(\mathrm{~d} x)\right)$. This yields (3.8)-(3.9) for $s=0$, and hence for all $s \in[0, T]$ by Remark 1.2(ii). Thus, the implication " $\Rightarrow$ " is shown, and the proof is complete. 
4.3. Proof of Proposition 3.14. It is clear that $\rho$ satisfies translation invariance, positive homogeneity and subadditivity.

Let us show that $\rho$ is not monotone by finding a Radon-Nikodym derivative in the dual representative set $S^{D}$ which takes values smaller than -1 on a nonzero set.

By Theorem 3.12, the set $C^{D}=\left(C_{t}^{D}\right)_{t \in[0, T]}$ is convex, closed, contains zero and has nonempty interior [for fixed $(t, \omega)$ ]. Furthermore, $C^{D}$ satisfies the dual representation in (3.8) with a bounded set $\mathcal{S}^{D}$ given in terms of $C^{D}$ by

$$
\mathcal{S}^{D}=\left\{\xi \in \mathcal{Q} \mid\left(H_{t}^{\xi}, \tilde{H}_{t}^{\xi}\right) \in C_{t}^{D} \text { for all } t \in[0, T]\right\} .
$$

As $C^{D}$ has nonempty interior, contains zero and $\partial C_{t}^{D}$ is deterministic, there exists a, say 1-dimensional, positive deterministic process $\left(\mu_{t}, \mathbf{0}\right) \in \partial C_{t}^{D}$ with $\mathbf{0}=(0, \ldots, 0) \in \mathbb{R}^{d}$. As $C_{t}^{D}$ is closed clearly $\left(\mu_{t}, \mathbf{0}\right) \in C_{t}^{D}$. We set for fixed $M>0: \lambda_{t}:=\frac{\min \left(\left|\mu_{t}\right|, M\right)}{\left|\mu_{t}\right|} \in(0,1]$ and $\hat{\mu}_{t}:=\lambda_{t} \mu_{t}+\left(1-\lambda_{t}\right) 0$. Note that $\hat{\mu}$ is bounded and positive by the definition of $\lambda_{t}$. Furthermore, $\left(\hat{\mu}_{t}, \mathbf{0}\right) \in C_{t}^{D}$ for every $t$ by the convexity of $C_{t}^{D}$. Define the Radon-Nikodym derivative $\xi:=(\hat{\mu} \cdot W)_{T}$. By definition $\xi$ has a normal distribution with nonzero variance $\int_{0}^{T} \hat{\mu}_{s}^{2} \mathrm{~d} s$ and by (4.11) $\xi \in \mathcal{S}^{D}$. In particular, $\xi$ takes values smaller than minus one with positive probability. As $\xi \in \mathcal{S}^{D}$ it follows that $\rho$ defined above is not monotone.

5. Dynamic mean-deviation portfolio optimization. We turn next to the stochastic optimization problem of identifying a dynamic portfolio allocation strategy that maximises the sum of the expected return and a penalty for its riskiness given in terms of a dynamic deviation measure of the final wealth achieved under this allocation strategy. Throughout this section, we impose the following conditions.

Assumption 5.1. (i) The Lévy measure $v$ is such that $v\left(\left\{x \in \mathbb{R}^{k} \backslash\{0\}\right.\right.$ : $\left.\left.\min _{i=1, \ldots, k} x_{i} \leq-1\right\}\right)=0$, and

$$
v_{2}:=\int_{\mathbb{R}^{k} \backslash\{0\}}|x|^{2} v(\mathrm{~d} x)<\infty .
$$

(ii) $D$ is a $g$-deviation measure with nonrandom, time-independent driver $\hat{g}$ : $\mathbb{R}^{d} \times L^{2}(v(\mathrm{~d} x)) \rightarrow \mathbb{R}_{+}$.

Under (5.1), $L=\left(L_{t}^{1}, \ldots, L_{t}^{k}\right)_{t \in[0, T]}^{\top}$ with $L_{t}^{j}=\int_{[0, t] \times \mathbb{R}^{k} \backslash\{0\}} x_{j} \tilde{N}(\mathrm{~d} s \times \mathrm{d} x), j=$ $1, \ldots, k$, where $x_{j}$ is the $j$ th coordinate of $x \in \mathbb{R}^{k}$, is a vector of pure-jump $\left(\mathcal{F}_{t}\right)$ martingales.

The financial market that we consider consists of a bank-account that pays interest at a fixed rate $r \geq 0$ and $n$ risky stocks (with $1 \leq n \leq \min \{d, k\}$ ) with price processes $S^{i}=\left(S_{t}^{i}\right)_{t \in[0, T]}, i=1, \ldots, n$, satisfying the SDEs given by

$$
\frac{\mathrm{d} S_{t}^{i}}{S_{t-}^{i}}=\mu_{i} \mathrm{~d} t+\sum_{j=1}^{d} \sigma_{i j} \mathrm{~d} W_{t}^{j}+\sum_{j=1}^{k} \rho_{i j} \mathrm{~d} L_{t}^{j}, \quad t \in(0, T],
$$


where $S_{0}^{i}=s_{i} \in \mathbb{R}_{+} \backslash\{0\}, \mu_{i} \in \mathbb{R}, \sigma_{i j} \in \mathbb{R}_{+}$and $\rho_{i j} \in \mathbb{R}_{+}$such that $\sum_{j=1}^{k} \rho_{i j} \leq$ 1 denote the rates of appreciation, the volatilities and the jump-sensitivities. By $\pi=\left(\pi^{1}, \ldots, \pi^{n}\right)^{\top}$, we denote the dynamic allocation process that indicates the fraction of the total wealth that is invested in the stocks $1, \ldots, n$ [i.e., if $X^{\pi}(t-)$ denotes the wealth just before time $t, \pi_{i}(t) X^{\pi}(t-)$ is the cash amount invested in stock $i$ at time $t$ under allocation strategy $\pi$ ]. We adopt the standard frictionless setting (no transaction costs, infinitely divisible stocks, continuous trading, etc.) and restrict to the case that short-sales and borrowing are not permitted, by only considering allocation processes $\pi=\left(\pi_{t}\right)_{t \in[0, T]}$ that take values in the set

$$
\mathcal{B}=\left\{x \in \mathbb{R}^{1 \times n}: \min _{i=1, \ldots, n} x_{i} \geq 0, \sum_{i=1}^{n} x_{i} \leq 1\right\} .
$$

Such an allocation process $\pi$ is said to be admissible if (i) $\pi$ is predictable, (ii) the associated wealth process $X^{\pi}$ is nonnegative (i.e., $X^{\pi}$ satisfies the insolvency constraint $\inf _{t \in[0, T]} X_{t}^{\pi} \geq 0$ ) and (iii) $\pi$ is a self-financing portfolio such that $X^{\pi}$ satisfies the $\operatorname{SDE}\left[\right.$ with $\mu=\left(\mu_{1}, \ldots, \mu_{n}\right)^{\top}, \Sigma=\left(\sigma_{i j}\right) \in \mathbb{R}^{n \times d}$ and $R=\left(\rho_{i j}\right) \in \mathbb{R}^{n \times k}$ ] given by

$$
\frac{\mathrm{d} X_{t}^{\pi}}{X_{t-}^{\pi}}=\left[r+(\mu-r \mathbf{1})^{\top} \pi_{t}\right] \mathrm{d} t+\pi_{t}^{\top} \Sigma \mathrm{d} W_{t}+\pi_{t}^{\top} R \mathrm{~d} L_{t}, \quad t \in(0, T]
$$

with initial wealth $X_{0}^{\pi}=x \in \mathbb{R}_{+} \backslash\{0\}$, where $\mathbf{1} \in \mathbb{R}^{n \times 1}$ denotes the column vector of ones. We denote by $\Pi$ the collection of admissible allocation strategies and let $\gamma>0$ denote a risk-aversion parameter. To a given allocation strategy $\pi \in \Pi$, we associate the following dynamic performance criterion:

$$
J_{t}^{\pi}:=E\left[X_{T}^{\pi} \mid \mathcal{F}_{t}\right]-\gamma D_{t}\left(X_{T}^{\pi}\right), \quad t \in[0, T]
$$

Due to the fact that, unlike the conditional expectation, $D_{t}(X)$ is a nonlinear function of $X$, the dynamic programming principle is not satisfied for this objective. There is a growing literature exploring alternative solution approaches to dynamic optimization problems for which the dynamic programming principle is not applicable. One alternative dynamic solution concept is that of subgame-perfect Nash equilibrium - in such a game-theoretic approach the problem (5.4) may informally be seen as a (noncooperative) game with infinitely many players, one for each time $t$, which may be interpreted in terms of the changing preferences of one person over time; see Ekeland and Pirvu (2008) and Björk and Murgoci (2010) for background, and see Basak and Chabakauri (2010), Björk and Murgoci (2010), Wang and Forsyth (2011), Czichowsky (2013), Björk, Murgoci and Zhou (2014), Bensoussan et al. (2014) and references therein, for studies of dynamic meanvariance portfolio optimization problems. Following Ekeland and Pirvu (2008) and Björk and Murgoci (2010), we have the following formalisation of this equilibrium solution concept in our setting. 
DEFINITION 5.2. (i) An allocation strategy $\pi^{*} \in \Pi$ is an equilibrium policy for the dynamic mean-deviation problem with objective (5.4) if

$$
\liminf _{h \searrow 0} \frac{J_{t}^{\pi^{*}}-J_{t}^{\pi(h)}}{h} \geq 0
$$

for any $t \in[0, T)$ and any policy $\pi(h) \in \Pi$ satisfying, for some $\pi \in \Pi$,

$$
\pi(h)_{s}=\pi_{s} I_{[t, t+h)}(s)+\pi_{s}^{*} I_{[t+h, T]}(s), \quad s \in[t, T] .
$$

(ii) An equilibrium policy $\pi^{*}$ is of feedback type if, for some feedback function $\pi_{*}:[0, T] \times \mathbb{R}_{+} \rightarrow \mathcal{B}$ such that $(5.3)$ with $\pi_{t}$ replaced by $\pi_{*}\left(t, X_{t-}\right)$ has a unique solution $X^{*}=\left(X_{t}^{*}\right)_{t \in[0, T]}$, we have

$$
\pi_{t}^{*}=\pi_{*}\left(t, X_{t-}^{*}\right), \quad t \in[0, T],
$$

with $X_{0-}^{*}=X_{0}^{*}$.

For a given equilibrium policy $\pi^{*}=\left(\pi_{t}^{*}\right)_{t \in[0, T]}$ of feedback-type, we have by the Markov property that $J_{t}^{\pi^{*}}=V\left(t, X_{t}^{*}\right)$ and $\mathbb{E}\left[X_{T}^{\pi^{*}} \mid \mathcal{F}_{t}\right]=h\left(t, X_{t}^{*}\right), t \in[0, T]$, for some functions $V:[0, T] \times \mathbb{R}_{+} \rightarrow \mathbb{R}_{+}$and $h:[0, T] \times \mathbb{R}_{+} \rightarrow \mathbb{R}_{+}$. Furthermore, if $h$ is sufficiently regular (e.g., $h \in C^{1,2}\left([0, T] \times \mathbb{R}_{+}, \mathbb{R}_{+}\right)$and $h^{\prime} \equiv \frac{\partial h}{\partial x}$ is bounded) we find by an application of Itô's lemma that the representing pair of $X_{T}^{*}$ is given by

$$
\begin{aligned}
H_{s}^{X_{T}^{*}}=a^{*, h}\left(s, X_{s-}^{*}\right), \quad \tilde{H}_{S}^{X_{T}^{*}}(y)=b^{*, h}\left(s, X_{s-}^{*}, y\right), & \\
& s \in[0, T], y \in \mathbb{R}^{k} \backslash\{0\},
\end{aligned}
$$

with

$$
\begin{aligned}
a^{*, h}(s, x) & :=h^{\prime}(s, x) x \pi_{*}(s, x)^{\top} \Sigma, \\
b^{*, h}(s, x, y) & :=h\left(s, x+x \pi_{*}(s, x)^{\top} R y\right)-h(s, x),
\end{aligned}
$$

so that $D_{t}\left(X_{T}^{*}\right), t \in[0, T]$, takes the form $D_{t}\left(X_{T}^{*}\right)=\tilde{D}_{t, X_{t}^{*}}\left(X_{T}^{*}\right)$, where

$$
\begin{aligned}
\tilde{D}_{t, x}\left(X_{T}^{*}\right)=\mathbb{E}_{t, x}\left[\int_{t}^{T} \hat{g}\left(a^{*, h}\left(s, X_{s-}^{*}\right), b^{*, h}\left(s, X_{s-}^{*}, \cdot\right)\right) \mathrm{d} s\right] & \\
& (t, x) \in[0, T] \times \mathbb{R}_{+},
\end{aligned}
$$

with $\mathbb{E}_{t, x}[\cdot]=\mathbb{E}\left[\cdot \mid X_{t}^{*}=x\right]$. To any vector $\pi \in \mathcal{B}$, we associate the operators $\mathcal{L}^{\pi}$ : $f \mapsto \mathcal{L}^{\pi} f$ and $\mathcal{G}^{\pi}: f \mapsto \mathcal{G}^{\pi} f$ that map $C^{0,2}\left([0, T] \times \mathbb{R}_{+}, \mathbb{R}\right)$ to $C^{0,0}\left(\mathbb{R}_{+}, \mathbb{R}\right)$ and are given by

$$
\begin{aligned}
& \mathcal{L}^{\pi} f(t, x)=\mu_{\pi} x f^{\prime}(t, x)+\frac{\sigma_{\pi}^{2}}{2} x^{2} f^{\prime \prime}(t, x)+\int_{\mathbb{R}^{k} \backslash\{0\}}\left[f\left(t, x+x \pi^{\top} R y\right)\right. \\
& \left.-f(t, x)-x \pi^{\top} R y f^{\prime}(t, x)\right] v(\mathrm{~d} y), \\
& \text { (5.8) } \mathcal{G}^{\pi} f(t, x)=\hat{g}\left(x f^{\prime}(t, x) \pi^{\top} \Sigma, \delta_{x \pi^{\top} R I} f(t, x)\right) \text {, }
\end{aligned}
$$


for $(t, x) \in[0, T] \times \mathbb{R}_{+}$, where $\delta_{y} f: \mathbb{R}_{+} \rightarrow \mathbb{R}$ and $I: \mathbb{R}^{k \times 1} \rightarrow \mathbb{R}^{k \times 1}$ are given by

$$
\delta_{y} f(x)=f(t, y+x)-f(t, x), \quad I(z)=z, \quad z \in \mathbb{R}^{k \times 1}, x \in \mathbb{R}_{+}, y \in \mathbb{R},
$$

and where

$$
\mu_{\pi}=r+(\mu-r \mathbf{1})^{\top} \pi, \quad \sigma_{\pi}^{2}=\pi^{\top} \Sigma \Sigma^{\top} \pi, \quad \pi \in \mathcal{B} .
$$

Given the form of the objective and Definition 5.2, we are led to consider the extended Hamilton-Jacobi-Bellman equation for a triplet $\left(\pi_{*}, V, h\right)$ of a feedback function $\pi_{*}$, the corresponding value function $V$ and auxiliary function $h$ given by (denoting $\dot{V}=\frac{\partial V}{\partial t}$ )

$$
\dot{V}(t, x)+\sup _{\pi \in \mathcal{B}}\left\{\mathcal{L}^{\pi} V(t, x)-\gamma \mathcal{G}^{\pi} h(t, x)\right\}=0,
$$

where, for any $t \in[0, T]$ and $x \in \mathbb{R}_{+}, \pi_{*}(t, x)$ is a maximiser of the supermum in (5.9) (note that the continuity of $\mathcal{L}^{\pi} V(t, x)$ and $\mathcal{G}^{\pi} h(t, x)$ in $\pi$ for each fixed $t \in[0, T]$ and $x \in \mathbb{R}_{+} \backslash\{0\}$ in conjunction with the compactness of $\mathcal{B}$ guarantees that the maximum in (5.9) is attained).

We have the following verification result.

THEOREM 5.3. Let $\left(\pi_{*}, h, V\right)$ be a triplet satisfying the extended HJB equation (5.9)-(5.12), let $X^{*}$ be the unique solution of (5.3) with $\pi_{t}$ replaced by $\pi_{*}\left(t, X_{t-}\right)$ and define $\pi_{t}^{*}=\pi_{*}\left(t, X_{t-}^{*}\right), t \in[0, T]$. Assume $h, V \in C^{1,2}([0, T] \times$ $\left.\mathbb{R}_{+}, \mathbb{R}\right)$ with $h^{\prime}, V^{\prime}$ bounded and that $\pi^{*}=\left(\pi_{t}^{*}\right)_{t \in[0, T]} \in \Pi$. Then $\pi^{*}$ is an equilibrium policy of feedback-type and $h$ and $V$ are given by $V(t, x)=\mathbb{E}_{t, x}\left[X_{T}^{\pi^{*}}\right]-$ $\gamma \tilde{D}_{t, x}\left(X_{T}^{\pi^{*}}\right)$ and $h(t, x)=\mathbb{E}_{t, x}\left[X_{T}^{\pi^{*}}\right]$ for $(t, x) \in[0, T] \times \mathbb{R}_{+}$.

Proof. We first verify the stochastic representations. Let $\pi=\left(\pi_{s}\right)_{s \in[0, T]} \in$ $\Pi, t \in[0, T)$ and $\tau \in(0, T-t)$ be given and denote $\Xi^{\pi, V, h}(s, x):=(\dot{V}+$ $\left.\mathcal{L}^{\pi_{s}} V-\gamma \mathcal{G}^{\pi_{s}} h\right)(s, x), a^{\pi, V}(s, x):=V^{\prime}(s, x) x \pi_{s}^{\top} \Sigma$ and $b^{\pi, V}(s, x, y)=V(s, x+$ $\left.x \pi_{s}^{\top} R y\right)-V(s, x)$. An application of Itô's lemma to $V\left(t+\tau, X_{t+\tau}^{\pi}\right)$ shows that

$$
\begin{aligned}
V(t+ & \left.\tau, X_{t+\tau}^{\pi}\right)-V\left(t, X_{t}^{\pi}\right)-\gamma \int_{t}^{t+\tau} \mathcal{G}^{\pi_{s}} h\left(s, X_{s-}^{\pi}\right) \mathrm{d} s \\
= & \int_{t}^{t+\tau} \Xi^{\pi, V, h}\left(s, X_{s-}^{\pi}\right) \mathrm{d} s+\int_{t}^{t+\tau} a^{\pi, V}\left(s, X_{s-}^{\pi}\right) \mathrm{d} W_{s} \\
& +\int_{(t, t+\tau] \times \mathbb{R}^{k} \backslash\{0\}} b^{\pi, V}\left(s, X_{s-}^{\pi}, y\right) \tilde{N}(\mathrm{~d} s \times \mathrm{d} y) .
\end{aligned}
$$


Similarly, it follows $h\left(t+\tau, X_{t+\tau}^{\pi}\right)$ satisfies (5.13) with $V, \mathcal{G}^{\pi_{s}} h$ and $\Xi^{\pi, V, h}$ replaced by $h, 0$ and $\Xi^{\pi, h, 0}$, respectively. In particular, choosing $\pi$ equal to $\pi^{*}$, and taking expectations, the three terms on the right-hand side of (5.13) vanish in view of (5.9), the fact that $\pi_{*}(t, x)$ is a maximiser in (5.9) and as the stochastic integrals are martingales (in view of the boundedness of $V^{\prime}, h^{\prime}$ and $\mathcal{B}$ ). Then, letting $\tau \nearrow T-t$ and using the boundary conditions (5.11), we obtain the asserted stochastic representations of $h$ and $V$.

Next, we turn to the proof that $\pi^{*}$ is an equilibrium solution. By an application of the tower-property of conditional expectation and (D6), we have for any $\pi \in \Pi$, $t \in[0, T)$ and $\tau \in(0, T-t)$ :

$$
\begin{aligned}
J_{t}^{\pi} & =\mathbb{E}\left[\mathbb{E}\left[X_{T}^{\pi} \mid \mathcal{F}_{t+\tau}\right] \mid \mathcal{F}_{t}\right]-\gamma \mathbb{E}\left[D_{t+\tau}\left(X_{T}^{\pi}\right) \mid \mathcal{F}_{t}\right]-\gamma D_{t}\left(\mathbb{E}\left[X_{T}^{\pi} \mid \mathcal{F}_{t+\tau}\right]\right) \\
& =\mathbb{E}\left[J_{t+\tau}^{\pi} \mid \mathcal{F}_{t}\right]-\gamma D_{t}\left(\mathbb{E}\left[X_{T}^{\pi} \mid \mathcal{F}_{t+\tau}\right]\right) .
\end{aligned}
$$

Fixing $\left(\epsilon_{n}\right)_{n}, \epsilon_{n} \searrow 0$, and strategies $\pi_{n}:=\pi\left(\epsilon_{n}\right) \in \Pi$ as in Definition 5.2 (with $\pi^{*}$ as asserted in the theorem) and noting that the Markov property (which is in force as $\pi^{*}$ is a feedback strategy) implies

$$
J_{t+\epsilon_{n}}^{\pi_{n}}=V\left(t+\epsilon_{n}, X_{t+\epsilon_{n}}^{\pi_{n}}\right), \quad \mathbb{E}\left[X_{T}^{\pi_{n}} \mid \mathcal{F}_{t+\epsilon_{n}}\right]=h\left(t+\epsilon_{n}, X_{t+\epsilon_{n}}^{\pi_{n}}\right),
$$

and that (5.15) remains valid with $\pi_{n}$ replaced by $\pi^{*}$, we have from (5.13) and (5.14) and the fact $D_{t}\left(h\left(t+\epsilon_{n}, X_{t+\epsilon_{n}}^{\pi_{n}}\right)\right)=\mathbb{E}\left[\int_{t}^{t+\epsilon_{n}} \hat{g}\left(a_{s}^{\pi_{n}, h}, b_{s}^{\pi_{n}, h}\right) \mathrm{d} s \mid \mathcal{F}_{t}\right]$ that

$$
J_{t}^{\pi^{*}}-J_{t}^{\pi_{n}}=\mathbb{E}\left[\int_{t}^{t+\epsilon_{n}}\left[\Xi^{\pi^{*}, V, h}\left(s, X_{s-}^{\pi^{*}}\right)-\Xi^{\pi_{n}, V, h}\left(s, X_{s-}^{\pi_{n}}\right)\right] \mathrm{d} s \mid \mathcal{F}_{t}\right] .
$$

Since $\Xi^{\pi_{*}(s, x), V, h}(s, x)=0$ and $\Xi^{\pi_{n}, V, h}(s, x) \leq 0$ for $s \in[0, T], x \in \mathbb{R}_{+}$, [by (5.9) and the fact that $\pi_{*}(t, x)$ is the maximiser in (5.9)] we have $\liminf _{n \rightarrow \infty}\left(J_{t}^{\pi^{*}}-\right.$ $\left.J_{t}^{\pi_{n}}\right) / \epsilon_{n} \geq 0$, and the proof is complete.

We next identify an explicit equilibrium policy for the mean-deviation portfolio optimization problem, under the following regularity assumption on $\Sigma, R$ and $\hat{g}$, assumed to be in force in the sequel.

ASSUMPTION 5.4. For some countable set $A$ and any $a \in\left[0, \gamma^{-1}\right] \backslash A$, the function $T_{a}: \mathcal{B} \rightarrow \mathbb{R}$ given by

$$
T_{a}(c):=a(\mu-r \mathbf{1})^{\top} c-\hat{g}\left(c^{\top} \Sigma, c^{\top} R I\right), \quad c \in \mathcal{B},
$$

achieves its maximum over $\partial B$ at a unique $c^{*} \in \partial \mathcal{B}{ }^{8}$

To define the optimal policy, we deploy the following auxiliary result.

\footnotetext{
${ }^{8} \partial \mathcal{B}$ denotes the boundary of $\mathcal{B}$, that is, $\partial B=\operatorname{cl}(\mathcal{B}) \backslash \operatorname{int}(\mathcal{B})$ where $\operatorname{cl}(\mathcal{B})$ and $\operatorname{int}(\mathcal{B})$ denote the closure and interior of $\mathcal{B}$.
} 
Lemma 5.5. For any $f:[0, T] \rightarrow \mathcal{B}$ denote by $A_{f}, d_{f}, b_{f}, F_{f}:[0, T] \rightarrow \mathbb{R}$ the functions given by

$$
\begin{aligned}
b_{f}(t) & :=\exp \left(\int_{t}^{T}\left\{r+(\mu-r \mathbf{1})^{\top} f(s)\right\} \mathrm{d} s\right), \\
d_{f}(t) & :=b_{f}(t) \int_{t}^{T} \hat{g}\left(f(s)^{\top} \Sigma, f(s)^{\top} R I\right) \mathrm{d} s, \\
A_{f}(t) & :=\gamma^{-1}-\left(b_{f}(t)\right)^{-1} d_{f}(t), \\
F_{f}(t) & :=A_{C_{f}}(t) \quad \text { with } \\
C_{f}(t) & := \begin{cases}\arg \sup _{c \in \partial \mathcal{B}}\left\{T_{f(t)}(c)\right\} & \text { if } f(t) \notin A, \\
\operatorname{Centroid}\left(\arg _{c \in \partial \mathcal{B}}\left\{T_{f(t)}(c)\right\}\right) & \text { if } f(t) \in A,\end{cases}
\end{aligned}
$$

where for any Borel set $A^{\prime} \subset \mathbb{R}^{d}$, Centroid $\left(A^{\prime}\right)$ is equal to the mean of $U \sim$ $\operatorname{Unif}\left(A^{\prime}\right)$. Then there exists a continuous nondecreasing function $a^{*}:[0, T] \rightarrow \mathbb{R}_{+}$ such that $a^{*}=F_{a^{*}}$.

The proof of Lemma 5.5 is provided below. With this result in hand, we identify an equilibrium policy as follows.

THEOREM 5.6. With $T_{a}(c)$ and $a^{*}$ given in (5.17) and in Lemma 5.5, we let $s(a):=\sup _{c \in \partial \mathcal{B}} T_{a}(c), a_{-}:=\sup \left\{a \in\left[0, \gamma^{-1}\right]: s(a) \leq 0\right\}$, and $t^{*}:=\sup \{t \in$ $\left.[0, T]: a^{*}(t) \leq a_{-}\right\}$(where $\left.\sup \varnothing:=-\infty\right)$.

(i) If $s(1 / \gamma) \leq 0$, then $\pi^{*} \equiv 0$ with value-function given by $V(t, x)=$ $x \exp (r(T-t))$ for $(t, x) \in[0, T] \times \mathbb{R}_{+}$.

(ii) If $s(1 / \gamma)>0$, define the function $C^{*}:[0, T] \rightarrow \mathcal{B}$ by

$$
C^{*}(t)= \begin{cases}C_{a^{*}(t)} & \text { if } t \in\left[t^{*} \vee 0,1\right], \\ 0 & \text { otherwise, }\end{cases}
$$

where $C_{a^{*}(t)}$ is given in (5.22) with $f=a^{*}$. Then $\pi^{*}=C^{*}$ is an equilibrium policy with value function given by $V(t, x)=x\left(b_{C^{*}}(t)-\gamma d_{C^{*}}(t)\right)$ for $(t, x) \in[0, T] \times$ $\mathbb{R}_{+}$, where $b_{C^{*}}$ and $d_{C^{*}}$ are given in (5.18) and (5.19) with $f=C^{*}$.

REMARK 5.7. Under the equilibrium policy $\pi^{*}$ given in Theorem 5.6, it is optimal to invest in the $n$ stocks according to the proportions $C^{*}=\left(C_{1}^{*}, \ldots, C_{n}^{*}\right)$ of the current wealth, which are nonrandom functions of $t$ only. Hence, it is optimal to invest at time $t$ an amount $X^{\pi^{*}}(t-) C_{i}^{*}(t)$ in stock $i, i=1, \ldots, n$.

Proof of ThEOREM 5.6. The proof consists in verifying that the triplet $\left(\pi_{*}, V, h\right)$, with $\pi_{*}$ and $V$ as stated and with $h:[0, T] \times \mathbb{R}_{+} \rightarrow \mathbb{R}$ given by 
$h(t, x)=x b_{C^{*}}(t)$, satisfies the extended HJB equation (5.9)-(5.12); the assertions then follow by an application of Theorem 5.3.

(i) Once we verify that the supremum in (5.9) is attained at $\pi_{*} \equiv 0$, it is easily checked that $V$ and $h$ are equal and satisfy (5.9)-(5.12), using that $g$ is positively homogeneous. To see that the former is the case, note that the left-hand side of (5.9) is equal to $x \exp (r(T-t))\left[-r+\gamma \sup _{c \in \mathcal{B}} T_{1 / \gamma}(c)\right]$; since $s(1 / \gamma) \leq 0$, the latter supremum is zero and it is attained at $c=0$ [as $\left.T_{1 / \gamma}(0)=0\right]$.

(ii) Assume for the moment that the supremum in (5.9) is attained at $\pi^{*}$. Then the positive homogeneity of $g$ and the fact (which is straightforward to verify) that functions $b_{C^{*}}$ and $d_{C^{*}}$ satisfy the system of equations

$$
\begin{aligned}
& \dot{b}+\left(r+\mu_{C^{*}}\right) b=0, \quad t \in[0, T), \quad b(T)=1, \\
& \dot{d}+\left(r+\mu_{C^{*}}\right) d+b \hat{g}\left(\left(C^{*}\right)^{\top} \Sigma,\left(C^{*}\right)^{\top} R I\right)=0, \quad t \in[0, T), \quad d(T)=0,
\end{aligned}
$$

where as before $I: \mathbb{R}^{k \times 1} \rightarrow \mathbb{R}^{k \times 1}$ is given by $I(y)=y$, imply that $h$ and $V$ satisfy (5.9)-(5.12).

Next, we verify that the supremum in (5.9) is attained at $\pi_{*}$. Inserting the forms of $h$ and $V$ and using that $\gamma \inf _{t \in[0, T]} b_{C^{*}}(t)>0$ we have for any $t \in[0, T]$ that

$$
\begin{aligned}
& \arg \sup _{\pi \in \mathcal{B}}\left\{\mathcal{L}^{\pi} V(t, x)-\gamma \mathcal{G}^{\pi} h(t, x)\right\} \\
& \quad=\arg \sup _{\pi \in \mathcal{B}}\left\{\mu_{\pi}\left(b_{C^{*}}(t)-\gamma d_{C^{*}}(t)\right)-\gamma b_{C^{*}}(t) \hat{g}\left(\pi^{\top} \Sigma, \pi^{\top} R I\right)\right\} \\
& \quad=\arg \sup _{\pi \in \mathcal{B}}\left\{\mu_{\pi} A_{C^{*}}(t)-\hat{g}\left(\pi^{\top} \Sigma, \pi^{\top} R I\right)\right\} .
\end{aligned}
$$

If $t \leq t^{*}$, then $A_{C^{*}}(t)=a_{-}$so that $s\left(A_{C^{*}}(t)\right) \leq 0$ and 0 is included in the argsup in (5.23), while if $t>t^{*}$, then $A_{C^{*}}(t)>a_{-}$and we have that $s\left(A_{C^{*}}(t)\right)=$ $\sup _{\pi \in \mathcal{B}}\left\{\left(\mu_{\pi}-r\right) A_{C^{*}}(t)-\hat{g}\left(\pi^{\top} \Sigma, \pi^{\top} R I\right)\right\}>0$ is attained at $\pi=C_{A_{C^{*}}}(t)=$ $C_{a^{*}(t)}=C^{*}(t)$.

PROOF OF LEMMA 5.5. The proof relies on an application of Schauder's fixed-point theorem ${ }^{9}$ to the map $F: \mathbb{A} \rightarrow C([0, T], \mathbb{R})$ given by $f \mapsto F_{f}$, where $\mathbb{A}$ denotes the set of continuous functions $f \in C([0, T], \mathbb{R})$ that are such that (a) $f(T)=\gamma^{-1}$ and (b) for all $s, t \in[0, T]$ with $s \leq t$ we have $f(t)-f(s) \in$ $\left[\chi_{-}(t-s), \chi_{+}(t-s)\right]$ where

$$
\chi_{+}:=\sup \left\{\hat{g}\left(c^{\top} \Sigma, c^{\top} R I\right): c \in \partial \mathcal{B}\right\}, \quad \chi_{-}:=\inf \left\{\hat{g}\left(c^{\top} \Sigma, c^{\top} R I\right): c \in \partial \mathcal{B}\right\} .
$$

We note that both $\chi_{+}$and $\chi_{-}$are strictly positive, by positivity of the driver function $\hat{g}$. It is straightforward to verify that $F$ maps $\mathbb{A}$ to $\mathbb{A}$ and that the set $\mathbb{A}$ is a nonempty, closed, bounded and convex subset of $C([0, T], \mathbb{R})$. Since $F$ is compact (as we prove below), Schauder's fixed-point theorem yields that there exists an element $a^{*} \in \mathbb{A}$ such that $a^{*}=F_{a^{*}}$.

\footnotetext{
${ }^{9}$ See, for example, Theorem 1.C in Zeidler (1995).
} 
We next prove that $F$ is compact by showing that (i) $F$ is continuous (with respect to the supremum norm on $[0, T]$ ) and (ii) the set $F(\mathbb{A})=\left\{F_{f}: f \in \mathbb{A}\right\}$ is relatively compact in $C([0, T], \mathbb{R})$.

(i) Let $\left(f_{n}\right)_{n} \subset \mathbb{A}$ converge to $f \in \mathbb{A}$ in the supremum-norm. Then we have that $T_{f_{n}(t)}(c) \rightarrow T_{f(t)}(c)$ as $n \rightarrow \infty$ uniformly in $t \in[0, T]$ for any $c \in \partial \mathcal{B}$, and $\sup _{c \in \partial \mathcal{B}} T_{f_{n}(t)}(c) \rightarrow \sup _{c \in \partial \mathcal{B}} T_{f(t)}(c)$ for any $t \in[0, T]$. As $\left(f_{n}\right)_{n}$ and $f$ are strictly monotone increasing and Assumption 5.4 is in force, we have for all but countably many $t$ that $T_{f_{n}(t)}(c)$ and $T_{f(t)}(c)$ attain their maxima over $\partial B$ at unique $c$. Thus, it follows that $\arg \sup _{c \in \partial \mathcal{B}} T_{f_{n}(t)}(c) \rightarrow \arg \sup _{c \in \partial \mathcal{B}} T_{f(t)}(c)$, for a.e. $t \in[0, T]$. Hence, by the dominated convergence theorem $F_{f_{n}}(t)=A_{C_{f_{n}}}(t) \rightarrow A_{C_{f}}(t)=$ $F_{f}(t)$ for any $t \in[0, T]$. Since the functions $A_{C_{f_{n}}}$ and $A_{C_{f}}$ are nondecreasing, the convergence $F_{f_{n}} \rightarrow F_{f}$ holds in the supremum norm.

(ii) Using the boundedness of $\mathcal{B}$ and the continuity of $\hat{g}$, it is straightforward to verify that the collection of functions $F(\mathbb{A})$ is equi-continuous. Hence, we have by an application of the Arzela-Ascoli theorem ${ }^{10}$ that for any sequence $\left(A^{(n)}\right)_{n} \subset F(\mathbb{A})$ there exists a continuous function $A^{*}:[0, T] \rightarrow \mathbb{R}$ such that, along a subsequence $\left(n_{k}\right),\left(A^{\left(n_{k}\right)}\right)_{k}$ converges uniformly to $A^{*}$, hence establishing that $F(\mathbb{A})$ is relatively compact.

EXAMPLE 5.8. (i) For driver function $\hat{g}=g_{1}$ (given in Example 2.10 with $\lambda=1)$ and for $a \in \mathbb{R}_{+}$we have that $T_{a}(c)$ in (5.17) is given by

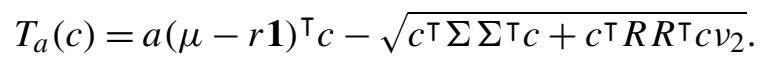

If $\Sigma \Sigma^{\top}+R R^{\top} v_{2}$ is invertible, then it is straightforward to verify that Assumption 5.4 is satisfied.

(ii) Let us identify explicitly the equilibrium portfolio allocation strategy given in Theorem 5.6 in the case the driver function $\hat{g}$ is as in part (i) and we have 2 risky assets $(n=2)$, whose dynamics we suppose are given by (5.2) with $d=k=$ $2, \mu_{1}>\mu_{2}>r, r \geq 0$ and $s_{12}:=\left(\Sigma^{2}+R^{2} v_{2}\right)_{12}<0$. In terms of $s_{i}^{2}:=\left(\Sigma^{2}+\right.$ $\left.R^{2} v_{2}\right)_{i i}, i=1,2$, let us denote

$$
\begin{aligned}
d_{+} & :=s_{1}^{2}+s_{2}^{2}-2 s_{12}, \quad e_{+}:=s_{12}-s_{2}^{2}, \\
c_{+}(a) & :=-\frac{e_{+}}{d_{+}}+\sqrt{\left(\frac{e_{+}}{d_{+}}\right)^{2}-\eta(a),} \quad \eta(a):=\frac{a^{2}\left(\mu_{1}-\mu_{2}\right)^{2} s_{2}^{2}-e_{+}^{2}}{d_{+}\left(a^{2}\left(\mu_{1}-\mu_{2}\right)^{2}-d_{+}\right)},
\end{aligned}
$$

for $a \in\left[0, \sqrt{d_{+}} /\left(\mu_{1}-\mu_{2}\right)\right)$. By convexity of $g$, it follows that the supremum of $\tilde{T}(c):=T_{a}((c, 1-c))=a\left(\mu_{2}-r\right)+a\left(\mu_{1}-\mu_{2}\right) c-\sqrt{d_{+} c^{2}+2 e_{+} c+s_{2}^{2}}$ over $c \in \mathbb{R}$ is attained at the $c$ satisfying $\tilde{T}^{\prime}(c)=0 \Leftrightarrow c=c_{+}(a)$ and we have

$$
\tilde{T}^{\prime}(1)>0 \Leftrightarrow a>a_{+}:=\frac{1}{\mu_{1}-\mu_{2}}\left(\frac{s_{1}^{2}-s_{12}}{\sqrt{s_{1}^{2}}}\right) .
$$

\footnotetext{
${ }^{10}$ See, for example, page 35 in Zeidler (1995).
} 
As a consequence, the equilibrium allocation strategy $\pi^{*}=\left(\pi_{t}^{*}\right)_{t \in[0, T]}$ in Theorem 5.6 is given as follows:

$$
\pi_{t}^{*}=C^{*}(t)= \begin{cases}(1,0) & \text { if } a_{*}(t)>a_{-} \vee a_{+}, \\ \left(c_{+}\left(a_{*}(t)\right), 1-c_{+}\left(a_{*}(t)\right)\right) & \text { if } a_{-}<a_{*}(t) \leq a_{-} \vee a_{+}, \\ (0,0) & \text { if } a_{*}(t) \leq a_{-},\end{cases}
$$

where $a_{-}$and $a_{*}(t)$ are as in Theorem 5.6. Hence, if the risk-aversion parameter $\gamma$ is sufficiently small and/or $t$ is sufficiently close to the horizon $T$, the equilibrium strategy is to be fully invested in risky asset 1 , which has the highest expected return; at times $t$ further away from the horizon or for higher risk-aversion parameter, the dynamic deviation penalty term starts to play a more important role and the policy is to invest part of the wealth into asset 2 , while if $\gamma$ is sufficiently large or $t$ is sufficiently small, the equilibrium strategy is to invest all the wealth in the bank account.

(iii) Restricting next to the case of a single risky asset $(n=1)$ with $d=k=1$, $\mu:=\mu_{1}>r$, we find by a direct calculation that the value function $V$ in Theorem 5.6 and he auxiliary function $h$ are explicitly given in terms of

$$
t^{*}=\left(T+\frac{1}{\mu-r}-\frac{1}{\gamma \sqrt{\Sigma^{2}+R^{2} \nu_{2}}}\right) \wedge T
$$

by $V(t, x)=V\left(t^{*} \wedge T, x \exp \left\{r\left(t^{*} \wedge T-t\right)\right\}\right)$ and $h(t, x)=h\left(t^{*} \wedge T, x \exp \left\{r\left(t^{*} \wedge\right.\right.\right.$ $T-t)\})$ for $t \in\left[0, t^{*} \wedge T\right)$ and

$$
\begin{aligned}
& V(t, x)=h(t, x)\left[1-(T-t) \gamma \sqrt{\Sigma^{2}+R^{2} v_{2}}\right] \\
& h(t, x)=x \exp \{\mu(T-t)\}, \quad t \in\left[t^{*} \wedge T, T\right],
\end{aligned}
$$

where the equilibrium policy $\pi^{*}$ is given by

$$
\begin{aligned}
\pi_{t}^{*}= & C^{*}(t) \\
= & \left\{\begin{array}{cl}
1 & \text { if } a(t)=\frac{1}{\gamma} \frac{1}{1+(\mu-r)(T-t)}>\frac{\sqrt{\Sigma^{2}+R^{2} v_{2}}}{\mu-r}=a_{-} \\
0 & \text { if } a(t) \leq a_{-} \Leftrightarrow t \in\left[0, t^{*} \wedge T\right] .
\end{array}\right.
\end{aligned}
$$

To see that $\pi^{*}$ takes this form, we observe that $t \leq t^{*}$ holds precisely if $\left(\mu-r-\gamma \sqrt{\Sigma^{2}+R^{2} v_{2}}\right)-(\mu-r) \gamma(T-t) \sqrt{\Sigma^{2}+R^{2} v_{2}} \leq 0 \Leftrightarrow 0 \in$ $\arg \sup _{\pi \in[0,1]}\left\{\left(\mathcal{L}^{\pi} V\right)(t, x)-\gamma\left(\mathcal{G}^{\pi} h\right)(t, x)\right\}$, where $\mathcal{L}^{\pi}$ and $\mathcal{G}^{\pi}$ are given in (5.7) and (5.8). 


\section{APPENDIX A: PROOFS OF LEMMAS 3.3, 3.4 AND 3.5}

ProOF OF LEMMA 3.3. (i) Let us first show that $C \mapsto \mu_{h, \tilde{h}}(C, \varnothing)$ constitutes a $\sigma$-finite measure. Clearly, $\mu_{h, \tilde{h}}(\cdot, \varnothing)$ is nonnegative and $\mu_{h, \tilde{h}}(\varnothing, \varnothing)=0$. Next, we verify that $C \mapsto \mu_{h, \tilde{h}}(C, \varnothing)$ is additive for disjoint sets of the form $C_{1}:=$ $\left(t_{1}, t_{2}\right] \times A$ and $C_{2}:=\left(t_{3}, t_{4}\right] \times B$ with $A \in \mathcal{F}_{t_{1}}$ and $B \in \mathcal{F}_{t_{3}}$. We consider first the case $t_{1} \leq t_{3} \leq t_{2} \leq t_{4}$ and $A \cap B=\varnothing$ (note that in this case $C_{1} \cap C_{2}=\varnothing$ ). By deploying Propositions 2.9 and 3.6 we note that $\mu_{h, \tilde{h}}\left(\left(\left(t_{1}, t_{2}\right] \times A\right) \cup\left(\left(t_{3}, t_{4}\right] \times\right.\right.$ $B), \varnothing)$ is equal to

$$
\begin{aligned}
D_{0}(( & \left.\left.I_{A} h \cdot W\right)_{t_{1}, t_{3}}+\left(I_{A \cup B} h \cdot W\right)_{t_{3}, t_{2}}+\left(I_{B} h \cdot W\right)_{t_{2}, t_{4}}\right) \\
= & \mathbb{E}\left[D_{t_{1}}\left(\left(I_{A} h \cdot W\right)_{t_{1}, t_{3}}\right)\right]+\mathbb{E}\left[D_{t_{3}}\left(\left(I_{A \cup B} h \cdot W\right)_{t_{3}, t_{2}}\right)\right] \\
& +\mathbb{E}\left[D_{t_{2}}\left(\left(I_{B} h \cdot W\right)_{t_{2}, t_{4}}\right)\right] \\
= & \mathbb{E}\left[D_{t_{1}}\left(\left(I_{A} h \cdot W\right)_{t_{1}, t_{3}}\right)\right]+\mathbb{E}\left[D_{t_{3}}\left(\left(I_{A} h \cdot W\right)_{t_{3}, t_{2}}\right)\right] \\
& \quad+\mathbb{E}\left[D_{t_{3}}\left(\left(I_{B} h \cdot W\right)_{t_{3}, t_{2}}\right)\right]+\mathbb{E}\left[D_{t_{2}}\left(\left(I_{B} h \cdot W\right)_{t_{2}, t_{4}}\right)\right] \\
= & D_{0}\left(\left(I_{A} h \cdot W\right)_{t_{1}, t_{2}}\right)+D_{0}\left(\left(I_{B} h \cdot W\right)_{t_{3}, t_{4}}\right),
\end{aligned}
$$

which is equal to $\mu_{h, \tilde{h}}\left(\left(t_{1}, t_{2}\right] \times A, \varnothing\right)+\mu_{h, \tilde{h}}\left(\left(t_{3}, t_{4}\right] \times B, \varnothing\right)$. The cases $t_{1} \leq$ $t_{2}<t_{3} \leq t_{4}$ and $t_{1} \leq t_{3} \leq t_{4} \leq t_{2}$ may be verified in a similar manner. Thus, we may conclude that $\mu_{h, \tilde{h}}$ is additive on disjoint sets of the form $\left(t_{1}, t_{2}\right] \times A$ and $\left(t_{3}, t_{4}\right] \times B$. As $D_{0}$ is continuous in $L^{2}\left(\mathcal{F}_{T}\right)$ [see Remark 1.2(iii)] and the collection of sets considered above is a semi-algebra generating the predictable $\sigma$-algebra it follows that $\mu_{h, \tilde{h}}(\cdot, \varnothing)$ is $\sigma$-finite. The proofs that $C \mapsto \mu_{h, \tilde{h}}(\varnothing, C)$ and $C \mapsto$ $\mu_{h, \tilde{h}}(C, C)$ are $\sigma$-finite measures are analogous, replacing in the equations above the term $h \cdot W$ by $\tilde{h} \cdot \tilde{N}$ and $(h \cdot W+\tilde{h} \cdot \tilde{N})$, respectively.

(ii) Define $C_{1}, C_{2}$ as in (i) and consider the case $t_{1} \leq t_{3} \leq t_{2} \leq t_{4}$ with general (not necessarily disjoint) $A \in \mathcal{F}_{t_{1}}$ and $B \in \mathcal{F}_{t_{3}}$. Expressing $X=I_{A}(h \cdot W)_{t_{1}, t_{2}}+$ $I_{B}(\tilde{h} \cdot \tilde{N})_{t_{3}, t_{4}}$ as the sum of martingale increments

$$
\begin{aligned}
X= & I_{A}(h \cdot W)_{t_{1}, t_{3}}+I_{A \backslash B}(h \cdot W)_{t_{3}, t_{2}}+I_{A \cap B}\left[(h \cdot W)_{t_{3}, t_{2}}+(\tilde{h} \cdot \tilde{N})_{t_{3}, t_{2}}\right] \\
& +I_{B \backslash A}(\tilde{h} \cdot \tilde{N})_{t_{3}, t_{2}}+I_{B}(\tilde{h} \cdot \tilde{N})_{t_{2}, t_{4}}
\end{aligned}
$$

and using Propositions 2.9 and 3.6 we have that $\mu_{h, \tilde{h}}\left(C_{1}, C_{2}\right)=D_{0}(X)$ is equal to

$$
\begin{aligned}
& \mathbb{E}\left[D_{t_{1}}\left(I_{A}(h \cdot W)_{t_{1}, t_{3}}\right)\right]+\mathbb{E}\left[D _ { t _ { 3 } } \left(I_{A \backslash B}(h \cdot W)_{t_{3}, t_{2}}\right.\right. \\
&+\left.\left.I_{A \cap B}\left[(h \cdot W)_{t_{3}, t_{2}}+(\tilde{h} \cdot \tilde{N})_{t_{3}, t_{2}}\right]+I_{B \backslash A}(\tilde{h} \cdot \tilde{N})_{t_{3}, t_{2}}\right)\right] \\
&+ \mathbb{E}\left[D_{t_{2}}\left(I_{B}(\tilde{h} \cdot \tilde{N})_{t_{2}, t_{4}}\right)\right] \\
&= \mathbb{E}\left[D_{t_{1}}\left(I_{A}(h \cdot W)_{t_{1}, t_{3}}\right)\right]+\mathbb{E}\left[D_{t_{3}}\left(I_{A \backslash B}(h \cdot W)_{t_{3}, t_{2}}\right)\right] \\
&+\mathbb{E}\left[D_{t_{3}}\left(I_{A \cap B}\left[(h \cdot W)_{t_{3}, t_{2}}+(\tilde{h} \cdot \tilde{N})_{t_{3}, t_{2}}\right]\right)\right] \\
&+\mathbb{E}\left[D_{t_{3}}\left(I_{B \backslash A}(\tilde{h} \cdot \tilde{N})_{t_{3}, t_{2}}\right)\right]+\mathbb{E}\left[D_{t_{2}}\left(I_{B}(\tilde{h} \cdot \tilde{N})_{t_{2}, t_{4}}\right)\right] .
\end{aligned}
$$


Thus, using Proposition 2.9 again we have

$$
\begin{aligned}
\mu_{h, \tilde{h}}\left(C_{1}, C_{2}\right)= & D_{0}\left(I_{A}(h \cdot W)_{t_{1}, t_{3}}+I_{A \backslash B}(h \cdot W)_{t_{3}, t_{2}}\right) \\
& +D_{0}\left(I_{B \cap A}\left[(h \cdot W)_{t_{3}, t_{2}}+(\tilde{h} \cdot \tilde{N})_{t_{3}, t_{2}}\right]\right) \\
& +D_{0}\left(I_{B \backslash A}(\tilde{h} \cdot \tilde{N})_{t_{3}, t_{2}}+I_{B}(\tilde{h} \cdot \tilde{N})_{t_{2}, t_{4}}\right) \\
= & \mu_{h, \tilde{h}}\left(C_{1} \backslash C_{2}, \varnothing\right)+\mu_{h, \tilde{h}}\left(C_{1} \cap C_{2}, C_{1} \cap C_{2}\right)+\mu_{h, \tilde{h}}\left(\varnothing, C_{2} \backslash C_{1}\right) .
\end{aligned}
$$

The cases $t_{1} \leq t_{2}<t_{3} \leq t_{4}$ and $t_{1} \leq t_{3} \leq t_{4} \leq t_{2}$ may be verified in a similar manner. By the continuity of $D_{0}$ [Remark 1.2(iii)] and monotone class arguments (by keeping first $C_{1}$ and then $C_{2}$ fixed), it follows that (3.1) holds for all predictable sets, as asserted.

PROOF OF LEMMA 3.4. First of all, note that the predictable $\sigma$-algebra is generated by countable many sets, say $A_{1}, A_{2}, \ldots$ Fix $n \in \mathbb{N}$ and denote $\mathcal{P}^{n}:=$ $\sigma\left(A_{1}, \ldots, A_{n}\right)$. By considering finer partitions, we may after relabeling assume without loss of generality that the $A_{i}$ are disjoint. Denote by $\eta$ the measure $\eta:=$ $\mathrm{d} \mathbb{P} \times \mathrm{d} t$ on $(\Omega \times[0, T], \mathcal{P})$ and let $R_{h, \tilde{h}}^{n}=E_{\eta}\left[R_{h, \tilde{h}} \mid \mathcal{P}^{n}\right] .{ }^{11}$ Since the filtration is generated by the disjoint sets $A_{1}, A_{2}, \ldots, A_{n}$, it is standard to note that

$$
R_{h, \tilde{h}}^{n}(s, \omega)=\sum_{i: v\left(A_{i}\right) \neq 0} \frac{I_{A_{i}}(s, \omega)}{\eta\left(A_{i}\right)} \mu_{h, \tilde{h}}\left(A_{i}, A_{i}\right) \quad \text { for } \mathrm{d} \mathbb{P} \times \mathrm{d} s \text { a.e. }(s, \omega)
$$

By possibly modifying $R_{h, \tilde{h}}^{n}$ on a zero-set, we may assume that (A.1) holds for all $(s, \omega) \in[0, T] \times \Omega$. It follows from (A.1) and the convexity and positive homogeneity of $(h, \tilde{h}) \rightarrow \mu_{h, \tilde{h}}\left(A_{i}, A_{i}\right)$ that, for all fixed $(s, \omega), R_{h, \tilde{h}}^{n}(s, \omega)$ is convex and positively homogeneous in $(h, \tilde{h})$. Furthermore, we claim that $\left|R_{h, \tilde{h}}^{n}\right| \leq g_{\lambda}(h, \tilde{h})$. Suppose this were not the case, that is, for some $(h, \tilde{h})$ and $A_{i},\left|R_{h, \tilde{h}}^{n}\right|>g_{\lambda}(h, \tilde{h})$ for all $(s, \omega) \in A_{i}$. Then we would have for $X=(H \cdot W)_{T}+(\tilde{H} \cdot \tilde{N})_{T}$ with $H_{s}=h I_{A_{i}}$ and $\tilde{H}_{s}=\tilde{h} I_{A_{i}}$ that $D_{0}(X)=\mu_{h, \tilde{h}}\left(A_{i}, A_{i}\right)=\mathbb{E}\left[\int_{0}^{T} I_{A_{i}}(s) R_{h, \tilde{h}}(s) \mathrm{d} s\right]$ satisfies

$$
D_{0}(X)>\mathbb{E}\left[\int_{0}^{T} I_{A_{i}}(s) g_{\lambda}(h, \tilde{h}) \mathrm{d} s\right]=\mathbb{E}\left[\int_{0}^{T} g_{\lambda}\left(H_{s}, \tilde{H}_{s}\right) \mathrm{d} s\right]=\bar{D}_{0}^{\lambda}(X),
$$

which is in contradiction with the fact that $D$ is $\lambda$-dominated.

Since $\mathcal{P}^{n}$ is an increasing sequence of $\sigma$-algebras with $\bigcup_{n=1}^{\infty} \mathcal{P}^{n}=\mathcal{P}$ it follows from the martingale convergence theorem that $R_{h, \tilde{h}}^{n}(t, \omega)=E_{\eta}\left[R_{h, \tilde{h}} \mid \mathcal{P}^{n}\right](t, \omega)$ converges to $E_{\eta}\left[R_{h, \tilde{h}} \mid \mathcal{P}\right](t, \omega)=R_{h, \tilde{h}}(t, \omega)$ for $\mathrm{dP} \times \mathrm{d} t$ a.e. $(t, \omega)$. This convergence only holds up to a zero set. On this zero set, we may set $R_{h, \tilde{h}}(t, \omega)$ equal

\footnotetext{
${ }^{11}$ Specifically, $R_{h, \tilde{h}}^{n}$ is the $\mathcal{P}^{n}$-measurable random variable satisfying $E_{\eta}\left[R_{h, \tilde{h}} U\right]=E_{\eta}\left[R_{h, \tilde{h}}^{n} U\right]$ for all bounded $\mathcal{P}^{n}$ random variables $U$, with $E_{\eta}[Z]=\int_{0}^{T} E[Z(s)] \mathrm{d} s$ for $Z \in L^{1}(\eta)$.
} 
to $\lim \sup _{n} R_{h, \tilde{h}}^{n}(t, \omega)$. Hence, this version of $R_{h, \tilde{h}}$ is dominated by $g_{\lambda}$ and is convex and positively homogeneous in $(h, \tilde{h})$ for every $(t, \omega) \in[0, T] \times \Omega$ as the limit of convex and positively homogeneous functions. The asserted continuity follows since every convex function that is locally bounded is continuous [see Theorem 2.2.9 in Zălinescu (2002)].

PROOF OF LEMMA 3.5. We split the proof in four steps.

Step 1: For $X=\left(\left(h I_{C_{1}}\right) \cdot W\right)_{T}+\left(\left(\tilde{h} I_{C_{2}}\right) \cdot \tilde{N}_{T}\right)$ for $(h, \tilde{h}) \in \mathbb{R} \times L^{2}(v(\mathrm{~d} x))$ and $C_{1}, C_{2} \in \mathcal{P}$, we find by using $g(t, \omega, 0,0)=0$ that $D_{0}^{g}(X)=$ $\mathbb{E}\left[\int_{0}^{T} g\left(s, h I_{C_{1}}(s), \tilde{h} I_{C_{2}}(s)\right) \mathrm{d} s\right]$ is equal to

$$
\mathbb{E}\left[\int_{0}^{T} I_{C_{1} \backslash C_{2}}(s) g(s, h, 0) \mathrm{d} s\right]+\mathbb{E}\left[\int_{0}^{T} I_{C_{2} \backslash C_{1}}(s) g(s, 0, \tilde{h}) \mathrm{d} s\right]
$$

$(\mathrm{A} .2)+\mathbb{E}\left[\int_{0}^{T} I_{C_{1} \cap C_{2}}(s) g(s, h, \tilde{h}) \mathrm{d} s\right]$

$$
=\mu_{h, \tilde{h}}\left(C_{1} \backslash C_{2}, \varnothing\right)+\mu_{h, \tilde{h}}\left(\varnothing, C_{2} \backslash C_{1}\right)+\mu_{h, \tilde{h}}\left(C_{1} \cap C_{2}, C_{1} \cap C_{2}\right),
$$

which is by (3.1) equal to $\mu_{h, \tilde{h}}\left(C_{1}, C_{2}\right)=D_{0}(X)$ [note that we only have to integrate over $C_{1} \cup C_{2}$ as $\left.g(t, \omega, 0,0)=0\right]$.

Step 2: Fix $t_{i}, t_{i+1} \in[0, T]$ with $t_{i}<t_{i+1}$ and let $X=\left(\left(h_{i} I_{\left(t_{i}, t_{i+1}\right]}\right) \cdot W\right)_{t_{i}, t_{i+1}}+$ $\left(\left(\tilde{h}_{i} I_{\left(t_{i}, t_{i+1}\right]}\right) \cdot \tilde{N}\right)_{t_{i}, t_{i+1}}$ with $h_{i}:=\sum_{j=1}^{m} c_{j} I_{A_{j}}, \tilde{h}_{i}=\sum_{j=1}^{m} \tilde{c}_{j} I_{A_{j}}$, and $c_{j} \in \mathbb{R}, \tilde{c}_{j} \in$ $L^{2}(v(\mathrm{~d} x))$, and disjoint sets $A_{j} \in \mathcal{F}_{t_{i}}, j=1, \ldots, m$, satisfying $\cup_{j} A_{j}=\Omega$ (we may assume w.l.o.g. that the $A_{j}$ are the same for $h$ and $\tilde{h}$ by setting some $c_{j}$ and $\tilde{c}_{j}$ equal to zero). By step 1 , denoting $\Delta W_{i+1}=W_{t_{i+1}}-W_{t_{i}}$,

$$
\begin{aligned}
& \mathbb{E}\left[\int_{t_{i}}^{t_{i+1}} g\left(s, I_{A_{j}} c_{j}, I_{A_{j}} \tilde{c}_{j}\right) \mathrm{d} s\right] \\
& \quad=D_{0}\left(I_{A_{j}} c_{j} \Delta W_{i+1}+\int_{\mathbb{R}^{k} \backslash\{0\}} I_{A_{j}} \tilde{c}_{j}(x) \tilde{N}\left(\left(t_{i}, t_{i+1}\right] \times \mathrm{d} x\right)\right) \\
& \quad=\mathbb{E}\left[D_{t_{i}}\left(I_{A_{j}} c_{j} \Delta W_{i+1}+\int_{\mathbb{R}^{k} \backslash\{0\}} I_{A_{j}} \tilde{c}_{j}(x) \tilde{N}\left(\left(t_{i}, t_{i+1}\right] \times \mathrm{d} x\right)\right)\right] .
\end{aligned}
$$

Hence, by Proposition $2.9 D_{0}(X)$ is equal to

$$
\begin{gathered}
\sum_{j=1}^{m} \mathbb{E}\left[D_{t_{i}}\left(I_{A_{j}} c_{j} \Delta W_{t_{i+1}}+\int_{\mathbb{R}^{k} \backslash\{0\}} I_{A_{j}} \tilde{c}_{j}(x) \tilde{N}\left(\left(t_{i}, t_{i+1}\right] \times \mathrm{d} x\right)\right)\right] \\
=\mathbb{E}\left[\sum_{j=1}^{m} \int_{t_{i}}^{t_{i+1}} g\left(s, I_{A_{j}} c_{j}, I_{A_{j}} \tilde{c}_{j}\right) \mathrm{d} s\right],
\end{gathered}
$$

which is equal to $\mathbb{E}\left[\int_{0}^{T} g\left(s, h_{s}, \tilde{h}_{s}\right) \mathrm{d} s \mid \mathcal{F}_{t}\right]=D_{0}^{g}(X)$. 
Step 3: Let $0 \leq t_{1}<\cdots<t_{n}=T$ be given. For simple functions $\underset{\tilde{N}}{X}=$ $\left(\left(\sum_{i=1}^{l} h_{i} I_{\left(t_{i}, t_{i+1}\right]}\right) \cdot W\right)_{T}+\left(\left(\sum_{i=1}^{l} \tilde{h}_{i} I_{\left(t_{i}, t_{i+1}\right]}\right) \cdot \tilde{N}\right)_{T}$ for $l \in \mathbb{N}$, with $h_{i}$ and $\tilde{h}_{i}$ as in step 2 we have by Proposition 2.9, step 2 and Proposition 2.4

$$
\begin{aligned}
D_{0}(X) & =\sum_{i=1}^{l} \mathbb{E}\left[D_{t_{i}}\left(\left(\left(h_{i} I_{\left(t_{i}, t_{i+1}\right]}\right) \cdot W\right)_{t_{i}, t_{i+1}}+\left(\left(\tilde{h}_{i} I_{\left(t_{i}, t_{i+1}\right]}\right) \cdot \tilde{N}\right)_{t_{i}, t_{i+1}}\right)\right] \\
& =\sum_{i=1}^{l} \mathbb{E}\left[D_{t_{i}}^{g}\left(\left(\left(h_{i} I_{\left(t_{i}, t_{i+1}\right]}\right) \cdot W\right)_{t_{i}, t_{i+1}}+\left(\left(\tilde{h}_{i} I_{\left(t_{i}, t_{i+1}\right]}\right) \cdot \tilde{N}\right)_{t_{i}, t_{i+1}}\right)\right]=D_{0}^{g}(X) .
\end{aligned}
$$

Hence, we have $D_{0}(X)=D_{0}^{g}(X)$ for all simple functions $X$.

Step 4: That $D_{0}(X)=D_{0}^{g}(X)$ not only for simple functions but also for general $X \in L^{2}\left(\mathcal{F}_{T}\right)$ follows by the continuity of $D_{0}^{g}$ and $D_{0}$ in Lemma 2.5 (note that $g$ is of linear growth) and Remark 1.2(iii).

\section{APPENDIX B: PROOFS OF PROPOSITIONS 4.3, 4.4 AND 3.14}

Proof of Proposition 4.3. Let $\xi \in L^{2}\left(\mathcal{F}_{T}\right)$ and $t \in[0, T]$. For brevity, we denote throughout the proof $\mathcal{S}=\mathcal{S}^{D}, \mathcal{S}_{t}=\mathcal{S}_{D_{t}}$ and $\mathcal{S}_{t, T}=\mathcal{S}_{t, T}^{D}$. As it is clear that $\mathcal{S} \cap \mathcal{Q}_{\mathcal{F}_{t}}=\mathcal{S}_{t, T}$ (noting that $\mathcal{S}_{t, T} \subset \mathcal{Q}_{\mathcal{F}_{t}}$ ), the remainder of the proof is concerned with showing that the sets $\mathcal{S} \cap \mathcal{Q}_{\mathcal{F}_{t}}$ and $\mathcal{S}_{t}$ are equal.

Noting that $\mathbb{E}\left[D_{t}(X)\right] \leq D_{0}(X)$ [by (D6)], recalling (4.2) and deploying (D6), (D1) and the fact that $L^{2}\left(\mathcal{F}_{T}\right)$ is directed, we have for $\xi \in \mathcal{S}_{t} \subset \mathcal{Q}_{\mathcal{F}_{t}}$

$$
\begin{aligned}
J_{\mathcal{S}}(\xi) & =\sup _{X \in L^{2}\left(\mathcal{F}_{T}\right)}\left\{\mathbb{E}[\xi X]-D_{0}(X)\right\} \leq \sup _{X \in L^{2}\left(\mathcal{F}_{T}\right)}\left\{\mathbb{E}[\xi X]-\mathbb{E}\left[D_{t}(X)\right]\right\} \\
& =\sup _{X \in L^{2}\left(\mathcal{F}_{T}\right)} \mathbb{E}\left[\mathbb{E}\left[\xi X \mid \mathcal{F}_{t}\right]-D_{t}(X)\right] \\
& =\mathbb{E}\left[\operatorname{ess} \sup _{X \in L^{2}\left(\mathcal{F}_{T}\right)}\left\{\mathbb{E}\left[\xi X \mid \mathcal{F}_{t}\right]-D_{t}(X)\right\}\right]=0,
\end{aligned}
$$

where in the last equality we used (4.2). As $J_{\mathcal{S}}(\xi)$ is either zero or infinity it follows from the previous display that $J_{\mathcal{S}}(\xi)=0$ implying that $\xi \in \mathcal{S}$ and thus $\xi \in \mathcal{S} \cap \mathcal{Q}_{\mathcal{F}_{t}}$. This shows $\mathcal{S}_{t} \subset \mathcal{S} \cap \mathcal{Q}_{\mathcal{F}_{t}}$.

On the other hand, if $\xi \in \mathcal{S}_{t}^{c}:=L^{2}\left(\mathcal{F}_{T}\right) \backslash \mathcal{S}_{t}$ then we have either (a) $\xi \in$ $\left(L^{2}\left(\mathcal{F}_{T}\right) \backslash \mathcal{Q}_{\mathcal{F}_{t}}\right) \cap \mathcal{S}_{t}^{c}$ or (b) $\xi \in \mathcal{Q}_{\mathcal{F}_{t}} \cap \mathcal{S}_{t}^{c}$. In case (a), we have $\xi \notin \mathcal{S} \cap \mathcal{Q}_{\mathcal{F}_{t}}$, while in case (b) (4.2) in Proposition 4.1 yields that there exists $X^{\prime} \in L^{2}\left(\mathcal{F}_{T}\right)$ such that $\mathbb{E}\left[\xi X^{\prime} \mid \mathcal{F}_{t}\right]-D_{t}\left(X^{\prime}\right)>0$ on a nonzero set, say $A$. Hence by using (D6) and that $\xi \in \mathcal{Q}_{\mathcal{F}_{t}}$, we have [from (4.2) with $t=0$ ]

$$
\begin{aligned}
J_{\mathcal{S}}(\xi) & \geq \mathbb{E}\left[\xi I_{A} X^{\prime}\right]-\mathbb{E}\left[D_{t}\left(X^{\prime} I_{A}\right)\right] \\
& =\mathbb{E}\left[I_{A}\left(\xi X^{\prime}-D_{t}\left(X^{\prime}\right)\right)\right]=\mathbb{E}\left[I_{A}\left(\mathbb{E}\left[\xi X^{\prime} \mid \mathcal{F}_{t}\right]-D_{t}\left(X^{\prime}\right)\right)\right]>0 .
\end{aligned}
$$


Thus, $J_{\mathcal{S}}(\xi)=\infty$ and we have that $\xi \notin \mathcal{S} \cap \mathcal{Q}_{\mathcal{F}_{t}}$, also in case (b). Hence, $\mathcal{S}_{t}^{c} \subset$ $L^{2}\left(\mathcal{F}_{T}\right) \backslash\left(\mathcal{S} \cap \mathcal{Q}_{\mathcal{F}_{t}}\right)$. Combined with the inclusion derived in previous paragraph, this yields that $\mathcal{S}_{t}=\mathcal{S} \cap \mathcal{Q}_{\mathcal{F}_{t}}$.

Proof of Proposition 4.4. We first show " $\Rightarrow$ ". We only give the proof that (D6) holds for $s=0$ as the proof for $s \in(0, T]$ is analogous. Let $X \in L^{2}\left(\mathcal{F}_{T}\right)$ and $t \in[0, T]$. Denoting $\xi_{t}=\mathbb{E}\left[\xi \mid \mathcal{F}_{t}\right]$ and $\xi_{t, T}=\xi-\xi_{t}$ for $\xi \in L^{2}\left(\mathcal{F}_{T}\right)$ we have

$$
\begin{aligned}
D_{0}(X) & =\sup _{\xi \in \mathcal{S}} \mathbb{E}[\xi X] \\
& =\sup _{\xi \in \mathcal{S}} \mathbb{E}\left[\mathbb{E}\left[\xi_{t} X+\left(\xi-\xi_{t}\right) X \mid \mathcal{F}_{t}\right]\right] \\
& =\sup _{\xi=\xi_{t}+\xi_{t, T} \in \mathcal{S}_{0, t}+\mathcal{S}_{t, T}}\left\{\mathbb{E}\left[\xi_{t} X\right]+\mathbb{E}\left[\mathbb{E}\left[\xi_{t, T} X \mid \mathcal{F}_{t}\right]\right]\right\} \\
& =\sup _{\xi_{t} \in \mathcal{S}_{0, t}, \xi_{t, T} \in \mathcal{S}_{t, T}}\left\{\mathbb{E}\left[\xi_{t} X\right]+\mathbb{E}\left[\mathbb{E}\left[\xi_{t, T} X \mid \mathcal{F}_{t}\right]\right]\right\} .
\end{aligned}
$$

Hence, by the directedness of $\mathcal{S}_{t, T}$ (Remark 4.2) and Proposition 4.3, we obtain

$$
\begin{aligned}
& D_{0}(X)=\sup _{\xi_{t} \in \mathcal{S}_{0, t}} \mathbb{E}\left[\xi_{t} \mathbb{E}\left[X \mid \mathcal{F}_{t}\right]\right]+\sup _{\xi_{t, T} \in \mathcal{S}_{t, T}} \mathbb{E}\left[\mathbb{E}\left[\xi_{t, T} X \mid \mathcal{F}_{t}\right]\right] \\
& =\sup _{\xi \in \mathcal{S}} \mathbb{E}\left[\xi \mathbb{E}\left[X \mid \mathcal{F}_{t}\right]\right]+\mathbb{E}\left[\operatorname{\xi ss}_{\xi_{t, T} \in \mathcal{S}_{t, T}}^{\operatorname{esup}} \mathbb{E}\left[\xi_{t, T} X \mid \mathcal{F}_{t}\right]\right] \\
& =D_{0}\left(\mathbb{E}\left[X \mid \mathcal{F}_{t}\right]\right)+\mathbb{E}\left[\underset{\xi \in \mathcal{S} \cap \mathcal{Q}_{\mathcal{F}_{t}}}{\operatorname{ess} \sup _{t}} \mathbb{E}\left[\xi X \mid \mathcal{F}_{t}\right]\right] \\
& =D_{0}\left(\mathbb{E}\left[X \mid \mathcal{F}_{t}\right]\right)+\mathbb{E}\left[D_{t}(X)\right] \text {. }
\end{aligned}
$$

To see that we have " $\Leftarrow$ " suppose that $\xi^{1}, \xi^{2} \in \mathcal{S}$ such that $\xi_{t}^{1}+\left(\xi^{2}-\xi_{t}^{2}\right) \notin \mathcal{S}$ for some $t \in[0, T]$. Then by the Hahn-Banach theorem, there exists a random variable $X \in L^{2}\left(\mathcal{F}_{T}\right)$ such that we have

$$
E:=\mathbb{E}\left[\left(\xi_{t}^{1}+\left(\xi^{2}-\xi_{t}^{2}\right)\right) X\right]>\sup _{\xi \in \mathcal{S}} \mathbb{E}[\xi X]=D_{0}(X) .
$$

Using Proposition 4.3, we note $E=\mathbb{E}\left[\xi_{t}^{1} \mathbb{E}\left[X \mid \mathcal{F}_{t}\right]\right]+\mathbb{E}\left[\mathbb{E}\left[\left(\xi^{2}-\xi_{t}^{2}\right) X \mid \mathcal{F}_{t}\right]\right]$ may be bounded above by

$$
\begin{aligned}
D_{0}\left(\mathbb{E}\left[X \mid \mathcal{F}_{t}\right]\right)+\mathbb{E}\left[\underset{\xi \in \mathcal{S}_{t, T}}{\operatorname{ess} \sup } \mathbb{E}\left[\xi X \mid \mathcal{F}_{t}\right]\right] & =D_{0}\left(\mathbb{E}\left[X \mid \mathcal{F}_{t}\right]\right)+\mathbb{E}\left[D_{t}(X)\right] \\
& =D_{0}(X)
\end{aligned}
$$

This bound is a contradiction to (B.1), which proves the implication " $\Leftarrow$ ". 


\section{REFERENCES}

Artzner, P., Delbaen, F., Eber, J.-M. and Heath, D. (1999). Coherent measures of risk. Math. Finance 9 203-228. MR1850791

Artzner, P., Delbaen, F., Eber, J.-M., Heath, D. and Ku, H. (2007). Coherent multiperiod risk adjusted values and Bellman's principle. Ann. Oper. Res. 152 5-22. MR2303124

BARRIEU, P. and El KAROUi, N. (2005). Inf-convolution of risk measures and optimal risk transfer. Finance Stoch. 9 269-298. MR2211128

BARrieu, P. and El Karoui, N. (2009). Pricing, hedging and optimally designing derivatives via minimization of risk measures. In Indifference Pricing: Theory and Applications (R. Carmona, ed.). 77-146. Princeton Univ. Press, Princeton, NJ.

BAsak, S. and Chabakauri, G. (2010). Dynamic mean-variance asset allocation. Rev. Financ. Stud. 23 2970-3016.

Bensoussan, A., Wong, K. C., Yam, S. C. P. and YUnG, S. P. (2014). Time-consistent portfolio selection under short-selling prohibition: From discrete to continuous setting. SIAM J. Financial Math. 5 153-190. MR3174170

Bion-NAdAl, J. and Kervarec, M. (2012). Risk measuring under model uncertainty. Ann. Appl. Probab. 22 213-238. MR2932546

BJÖRK, T. and MURGOCI, A. (2010). A general theory of Markovian Time Inconsistent Stochastic Control Problems. Working paper, Stockholm School of Economics.

BJÖRK, T., MurgOCI, A. and Zhou, X. Y. (2014). Mean-variance portfolio optimization with state-dependent risk aversion. Math. Finance 24 1-24. MR3157686

BLACK, F. and SCHOLES, M. (1973). The pricing of options and corporate liabilities. J. Polit. Econ. 81 637-654. MR3363443

Chen, Z. and EPSTEIn, L. (2002). Ambiguity, risk, and asset returns in continuous time. Econometrica 70 1403-1443. MR1929974

Cheng, S., LiU, Y. and WAng, S. (2004). Progress in risk measurement. Adv. Model. Optim. 6 1-20. MR2304250

Cheridito, P. and KupPer, M. (2011). Composition of time-consistent dynamic monetary risk measures in discrete time. Int. J. Theor. Appl. Finance 14 137-162. MR2780781

Coquet, F., Hu, Y., Mémin, J. and Peng, S. (2002). Filtration-consistent nonlinear expectations and related $g$-expectations. Probab. Theory Related Fields 123 1-27. MR1906435

CzICHOWsKY, C. (2013). Time-consistent mean-variance portfolio selection in discrete and continuous time. Finance Stoch. 17 227-271. MR3038591

Delbaen, F. (2006). The structure of $\mathrm{m}$-stable sets and in particular of the set of risk neutral measures. In In Memoriam Paul-André Meyer: Séminaire de Probabilités XXXIX. Lecture Notes in Math. 1874 215-258. Springer, Berlin. MR2276899

Delbaen, F., Hu, Y. and BAO, X. (2011). Backward SDEs with superquadratic growth. Probab. Theory Related Fields 150 145-192. MR2800907

Delbaen, F., Peng, S. and Rosazza Gianin, E. (2010). Representation of the penalty term of dynamic concave utilities. Finance Stoch. 14 449-472. MR2670421

Durrett, R. (2010). Probability: Theory and Examples, 4th ed. Cambridge Series in Statistical and Probabilistic Mathematics 31. Cambridge Univ. Press, Cambridge. MR2722836

EKELAND, I. and PIRVU, T. A. (2008). Investment and consumption without commitment. Math. Financ. Econ. 2 57-86. MR2461340

El Karoui, N. and RavanElli, C. (2009). Cash subadditive risk measures and interest rate ambiguity. Math. Finance 19 561-590. MR2583520

Föllmer, H. and SchIED, A. (2011). Stochastic Finance: An Introduction in Discrete Time, 3rd extended ed. de Gruyter, Berlin. MR2779313 
Grechuk, B., Molyboha, A. and Zabarankin, M. (2009). Maximum entropy principle with general deviation measures. Math. Oper. Res. 34 445-467. MR2554068

Grechuk, B., Molyboha, A. and Zabarankin, M. (2013). Cooperative games with general deviation measures. Math. Finance 23 339-365. MR3034081

GRECHUK, B. and ZABARANKIN, M. (2014). Inverse portfolio problem with mean-deviation model. European J. Oper. Res. 234 481-490. MR3144737

Hu, Y., MA, J., PEnG, S. and YAO, S. (2008). Representation theorems for quadratic $F$-consistent nonlinear expectations. Stochastic Process. Appl. 118 1518-1551. MR2442369

JACOD, J. and Shiryaev, A. N. (2003). Limit Theorems for Stochastic Processes. Grundlehren der Mathematischen Wissenschaften [Fundamental Principles of Mathematical Sciences] 288. Springer, Berlin. MR0959133

JIANG, L. (2008). Convexity, translation invariance and subadditivity for $g$-expectations and related risk measures. Ann. Appl. Probab. 18 245-258. MR2380898

KLÖPPEL, S. and SCHWEIZER, M. (2007). Dynamic indifference valuation via convex risk measures. Math. Finance 17 599-627. MR2352907

Krätschmer, V., Ladkau, M., Laeven, R. A., Schoenmakers, J. and Stadje, M. (2015). Optimal stopping under drift and jump uncertainty. Preprint, available at https://sites.google.com/site/mstadje/.

LI, Z., ZENG, Y. and LAI, Y. (2012). Optimal time-consistent investment and reinsurance strategies for insurers under Heston's SV model. Insurance Math. Econom. 51 191-203. MR2928756

MÄRKERT, A. and SCHULTZ, R. (2005). On deviation measures in stochastic integer programming. Oper. Res. Lett. 33 441-449. MR2146607

Markowitz, H. M. (1952). Portfolio selection. J. Finance 7 77-91.

Pelsser, A. and Stadje, M. (2014). Time-consistent and market-consistent evaluations. Math. Finance 24 25-65. MR3157687

RIEDEl, F. (2004). Dynamic coherent risk measures. Stochastic Process. Appl. 112 185-200. MR2073410

Rockafellar, R. T., Uryasev, S. and Zabarankin, M. (2006a). Generalized deviations in risk analysis. Finance Stoch. 10 51-74. MR2212567

Rockafellar, R. T., Uryasev, S. and Zabarankin, M. (2006b). Optimality conditions in portfolio analysis with general deviation measures. Math. Program. 108 515-540. MR2238713

Rockafellar, R. T., Uryasev, S. P. and Zabarankin, M. (2006c). Master funds in portfolio analysis with general deviation measures. J. Bank. Financ. 30 743-778.

Rockafellar, R. T., Uryasev, S. P. and Zabarankin, M. (2007). Equilibrium with investors using a diversity of deviation measures. J. Bank. Financ. 31 3251-3268.

RosaZZA Gianin, E. (2006). Risk measures via $g$-expectations. Insurance Math. Econom. 39 1934. MR2241848

ROYER, M. (2006). Backward stochastic differential equations with jumps and related non-linear expectations. Stochastic Process. Appl. 116 1358-1376. MR2260739

RuszcZyński, A. and Shapiro, A. (2006). Conditional risk mappings. Math. Oper. Res. $31544-$ 561. MR2254423

Stoyanov, S. V., Rachev, S. T., Ortobelli, S. and Fabozzi, F. J. (2008). Relative deviation metrics and the problem of strategy replication. J. Bank. Financ. 32 199-206.

WANG, J. and ForSYTh, P. A. (2011). Continuous time mean variance asset allocation: A timeconsistent strategy. European J. Oper. Res. 209 184-201. MR2746866

ZăLinescu, C. (2002). Convex Analysis in General Vector Spaces. World Scientific, River Edge, NJ. MR1921556

ZeIDler, E. (1995). Applied Functional Analysis: Applications to Mathematical Physics. Applied Mathematical Sciences 108. Springer, New York. MR1347691 
DEPARTMENT OF MATHEMATICS

IMPERIAL COLLEGE LONDON

$6 \mathrm{M} 13$

HUXLEY BUILDING

South Kensington Campus

UNITED KINGDOM

E-MAIL: m.pistorius@imperial.ac.uk
FACULTY OF MATHEMATICS AND ECONOMICS UNIVERSITY OF ULM

HELMHOLTZSTRASSE 20

89069 ULM

GERMANY

E-MAIL: mitja.stadje@uni-ulm.de 